\title{
Beclin 2 negatively regulates innate immune signaling and tumor development
}

\author{
Motao Zhu, ${ }^{1,2,3,4}$ Guangtong Deng, ${ }^{3,5}$ Peng Tan, ${ }^{3}$ Changsheng Xing, ${ }^{1,2,3}$ Cuiping Guan, ${ }^{6}$ Chongming Jiang, ${ }^{3}$ Yinlong Zhang, ${ }^{4}$ \\ Bo Ning, ${ }^{3}$ Chaoran Li, ${ }^{3,5}$ Bingnan Yin, ${ }^{1,2,3,7}$ Kaifu Chen, ${ }^{8}$ Yuliang Zhao, ${ }^{4}$ Helen Y. Wang, ${ }^{1,2,3,7}$ Beth Levine, ${ }^{9,10}$ Guangjun Nie, ${ }^{4}$ \\ and Rong-Fu Wang ${ }^{1,2,3,7}$
}

\begin{abstract}
'Department of Medicine and ${ }^{2}$ Norris Comprehensive Cancer Center, Keck School of Medicine, University of Southern California, Los Angeles, California, USA. ${ }^{3}$ Center for Inflammation and Epigenetics, Houston Methodist Research Institute, Houston, Texas, USA. ${ }^{4}$ CAS Key Laboratory for Biomedical Effects of Nanomaterials and Nanosafety, CAS Center for Excellence in Nanoscience, National Center for Nanoscience and Technology of China, Beijing, China. ${ }^{5}$ Ceneral Surgery Department, Xiangya Hospital, Central South University, Changsha, China. ${ }^{6}$ Department of Dermatology, Third People's Hospital of Hangzhou, Hangzhou, China. 'Department of Pediatrics, Children's Hospital Los Angeles, Keck School of Medicine, University of Southern California, Los Angeles, California, USA. ${ }^{8}$ Center for Regenerative Medicine, Department of Cardiovascular Sciences, Houston Methodist Research Institute, Houston, Texas, USA. ${ }^{9}$ Center for Autophagy Research, University of Texas Southwestern Medical Center, Dallas, Texas, USA. ${ }^{10}$ Howard Hughes Medical Institute, Dallas, Texas, USA.
\end{abstract}

Beclin 2 plays a critical role in metabolic regulation and obesity, but its functions in innate immune signaling and cancer development remain largely unknown. Here, we identified Beclin 2 as a critical negative regulator of inflammation and lymphoma development. Mice with homozygous ablation of BCL2-interacting protein 2 (Becn2) developed splenomegaly and lymphadenopathy and markedly increased ERK1/2 and NF-кB signaling for proinflammatory cytokine production. Beclin 2 targeted the key signaling kinases MEKK3 and TAK1 for degradation through an ATC9A-dependent, but ATC16L/ Beclin 1/LC3-independent, autophagic pathway. Mechanistically, Beclin 2 recruited MEKK3 or TAK1 through ATC9A to form a complex (Beclin 2-ATC9A-MEKK3) on ATC9A ${ }^{+}$vesicles upon ULK1 activation. Beclin 2 further interacted with STX5 and STX6 to promote the fusion of MEKK3- or TAK1-associated ATC9A+ vesicles to phagophores for subsequent degradation. Importantly, Becn2-deficient mice had a markedly increased incidence of lymphoma development, with persistent STAT3 activation. Myeloid-specific ablation of MEKK3 (Map3k3) completely rescued the phenotypes (splenomegaly, higher amounts of proinflammatory cytokines, and cancer incidence) of Becn2-deficient mice. Hence, our findings have identified an important role of Beclin 2 in the negative regulation of innate immune signaling and tumor development through an ATC9Adependent, but ATC16L/Beclin 1/LC3-independent, autophagic pathway, thus providing a potential target for the treatment of inflammatory diseases and cancer.

\section{Introduction}

Autophagy is an essential cellular process for maintaining cell homeostasis and attenuating cell stresses through a "self-eating" mechanism. Many autophagy-related (ATG) proteins have been identified as functioning in autophagy to control physiological and pathological processes by breaking down misfolded or dysfunctional components for recycling $(1,2)$. Notably, growing evidence shows that ATG proteins could regulate immune responses through canonical macroautophagy, noncanonical macroautophagy, or autophagy-independent pathways (3-7). Beclin 2 (Becn2) has recently been identified as a homolog of Becn1 with both autophagy-dependent and -independent functions; it targets $G$ protein-coupled receptors (GPCRs) for degradation through the endosomal-lysosomal pathway (8). Heterozygous Becn2-KO mice develop obesity and insulin resistance due to excessive cannabinoid 1 receptor (CB1R) signaling (8).

Authorship note: $\mathrm{BL}$ is deceased.

Conflict of interest: The authors have declared that no conflict of interest exists. Copyright: () 2020, American Society for Clinical Investigation.

Submitted: September 9, 2019; Accepted: July 1, 2020; Published: August 31, 2020.

Reference information: J Clin Invest. 2020;130(10):5349-5369.

https://doi.org/10.1172/JCl133283.
Furthermore, monoallelic deletion of Becn2 significantly increases Kaposi's sarcoma-associated herpesvirus-induced (KSHV-induced) oncogenesis through elevation of KSHV GPCR signaling in ikGP$\mathrm{CR}^{+} \mathrm{BeCn2}^{+/-}$mice (9). Obesity-associated chronic inflammation plays a leading role in the pathogenesis of type II diabetes and cancer (10, 11). Despite the importance of autophagy and its related proteins in immunity and cancer development (12-14), the function and mechanisms of Beclin 2 in the regulation of innate immune signaling, autophagy, and cancer remain largely unknown.

Innate immune signaling pathways, including the $\mathrm{NF}-\kappa \mathrm{B}$, type I IFN, and inflammasome pathways, are activated through innate immune receptors, such as Toll-like receptors, RIG-I-like receptors, DNA sensors, and NOD-like receptors (15-17). Ligation of these immune receptors with pathogen-associated molecular patterns (PAMPs) and damage-associated molecular patterns (DAMPs) recruits key adaptor molecules to trigger downstream signaling pathways for the production of inflammatory cytokines (18). The regulatory functions of ATG proteins in innate immune signaling are crucial for the control of systemic inflammation. For instance, the ULK1/2 complex and Beclin 1 protect the host against viral infection $(19,20)$, while ATG16L1 suppresses endotoxin-induced inflammasome activation (21). Autophagy 
may also function as a tumor suppressor to inhibit cancer development by regulating innate immune signaling (14).

In this study, we describe the role of Beclin 2 as a negative regulator in the control of the ERK1/2 and NF- $\mathrm{KB}$ signaling pathways for suppressing inflammation and tumor development. Mice with homozygous ablation of Becn2 developed splenomegaly and lymphadenopathy and enhanced phosphorylation of ERK1/2 and NF- $\mathrm{KB}$ signaling in innate immune cells for proinflammatory cytokine productions. Mechanistically, Beclin 2 targeted mitogen-activated protein kinase kinase kinase 3 (MEKK3) and mitogen-activated protein kinase kinase kinase 7 (TAK1) for autophagic degradation through an ATG9A-dependent but ATG16L/ LC3/Beclin 1-independent pathway. Beclin 2 promoted the membrane fusion of TAK1/MEKK3-associated ATG9A ${ }^{+}$vesicles with phagophores through its interaction with STX5/STX6. Importantly, Becn2-deficient mice developed spontaneous lymphoma at a high incidence ( $13.2 \%)$ with persistent activation of STAT3 signaling by IL-6 and other cytokines. Our findings have identified an important role of Beclin 2 in the regulation of innate immune signaling and tumor development, thus providing a potential therapeutic target for the prevention and treatment of inflammatory diseases and cancer.

\section{Results}

Splenomegaly and lymphadenopathy in Becn2-deficient mice. To investigate the potential role of Beclin 2 in the immune system, we first analyzed human BECN2 expression in multiple organs and different cell types and found that human BECN2 was highly expressed in the thymus, lung, liver, and pancreas, particularly in PBMCs, DCs, T cells, and B cells (Figure 1A). Similarly, mouse Becn2 was also highly expressed in immune cells and lymphoid tissues, such as neutrophils, T cells, B cells, and spleen, compared with nonimmune tissues (Supplemental Figure 1A; supplemental material available online with this article; https://oi.org/10.1172/ JCI133283DS1). To assess the function of Beclin 2 in immune cells, we generated homozygous Becn2-KO mice despite the partial lethality of Becn2 ablation (8). Becn2 deficiency was validated by PCR using specific primers (Supplemental Figure 1B). We found that Becn2-KO mice developed splenomegaly and lymphadenopathy (Figure 1B). Histological examination revealed grossly disorganized architectures of spleens and lymph nodes in Becn2-KO mice, with complete loss of the marginal zone barrier (Figure 1C). Mild architecture difference was also found in the thymus of Becn2-KO mice compared with WT mice (Supplemental Figure 1C). Consistent with the splenomegaly and enlarged lymph nodes, we observed marked increases in the total numbers of splenocytes and lymphocytes from inguinal lymph nodes, particularly the $\mathrm{B}_{22} 2 \mathrm{O}^{+} \mathrm{B}$ cell and $\mathrm{CD}^{+} \mathrm{T}$ cell numbers, in Becn2-KO mice compared with WT mice (Figure 1, D-F). However, we did not observe significant differences in the subsets of either $\mathrm{T}$ cells or $\mathrm{B}$ cells between WT and Becn2-KO mice by CyTOF (mass cytometry) analyses (Supplemental Figure 1, D and E). There was no appreciable difference in macrophage/neutrophil populations in the spleens between WT and Becn2-KO mice (Supplemental Figure $1 F)$. These results suggest that loss of Beclin 2 results in splenomegaly and lymph node enlargement with grossly disorganized architectures and increased total lymphocyte populations.
Beclin 2 deficiency increases proinflammatory cytokine production through ERK and NF- $\kappa B$ signaling. We next determined the levels of proinflammatory cytokines in WT and Becn2-KO immune cells after TLR ligand stimulation. We found that Becn2deficient BM-derived DCs (BMDCs) and BM-derived macrophages (BMDMs) produced more IL-6, but not TNF- $\alpha$, than WT cells after LPS (a TLR4 ligand) treatment (Figure 2, A and B). LPS-primed DCs and macrophages from Becn2-deficient mice also produced more IL-1 $\beta$ than corresponding WT cells after adenosine triphosphate (ATP) treatment (Figure 2, A and B). Furthermore, Becn2deficient neutrophils produced significantly more TNF- $\alpha$, IL-6, and IL-1 $\beta$ than WT controls after LPS treatment (Figure 2C). Consistent with the cytokine production, RNA-Seq analyses showed increased expressions of IL- 6 and IL- $1 \beta$ as well as increased expression of other cytokines and chemokines in LPS-treated Becn2-KO BMDMs (Figure 2D). Ingenuity pathway analysis using RNA-Seq data further revealed that the TNF signaling, NOD-like receptor signaling, cytokine-cytokine receptor interaction, and chemokine signaling pathways were markedly increased in Becn2-KO macrophages compared with WT cells (Supplemental Figure 2A). To determine the specificity of the innate immune response, we examined proinflammatory cytokine expression following stimulation with poly(I:C) (a ligand of TLR3) and CpG oligonucleotides (a ligand of TLR9) and found that Becn2-deficient macrophages produced more IL-6, but not TNF- $\alpha$, than WT macrophages after poly(I:C) treatment (Supplemental Figure 2B). However, we did not observe any appreciable difference in IL- 6 or TNF- $\alpha$ production between Becn2-deficient and WT DCs after stimulation with CpG oligonucleotides (Supplemental Figure 2C). These results suggest that Beclin 2 deficiency negatively regulates IL-6, IL-1 $\beta$, or TNF- $\alpha$ production in a cell type- and TLR ligand-specific manner. Since TRIF is the adapter for both TLR3/TLR4 and type I IFN signaling, we examined the role of Beclin 2 in the type I IFN signaling pathway by detecting the IFN- $\beta$ production in BMDMs after vesicular stomatitis virus (VSV) infection or poly(dA:dT) treatment. We found no appreciable difference in IFN- $\beta$ production between WT and Becn2-KO macrophages (Supplemental Figure 2D). Consistently, no significant change in ISRE activity was detected in poly(I:C)- or poly(dA:dT)-stimulated 293T cells transfected with ISRE-luciferase reporter and increasing amounts of Beclin 2 expression plasmids (Supplemental Figure 2E), suggesting that Beclin 2 does not play a critical role in type I IFN signaling.

To substantiate these findings under physiological conditions, we determined the sensitivity of Becn2-KO mice to LPS-induced septic shock. After i.p. injection of LPS at $30 \mathrm{mg} / \mathrm{kg}$ body weight, Becn2-KO mice exhibited significantly shortened survival and rapidly died, within 34 hours, while $40 \%$ of WT counterparts survived over 40 hours (Figure 2E). Consistently, increased serum levels of IL- 6 and IL-1 $\beta$ were observed in Becn2-KO mice compared with WT mice after LPS treatment (Figure 2F). Notably, the serum levels of IL-6, but not other cytokines tested, were significantly elevated in Becn2-KO mice compared with those in WT mice even before LPS treatment (Figure $2 \mathrm{G}$ and Supplemental Figure 2F), suggesting higher spontaneous production (basal level) of IL-6 in Becn2-KO mice.

To understand the molecular mechanisms responsible for the elevated levels of proinflammatory cytokines in Becn2-deficient 
A

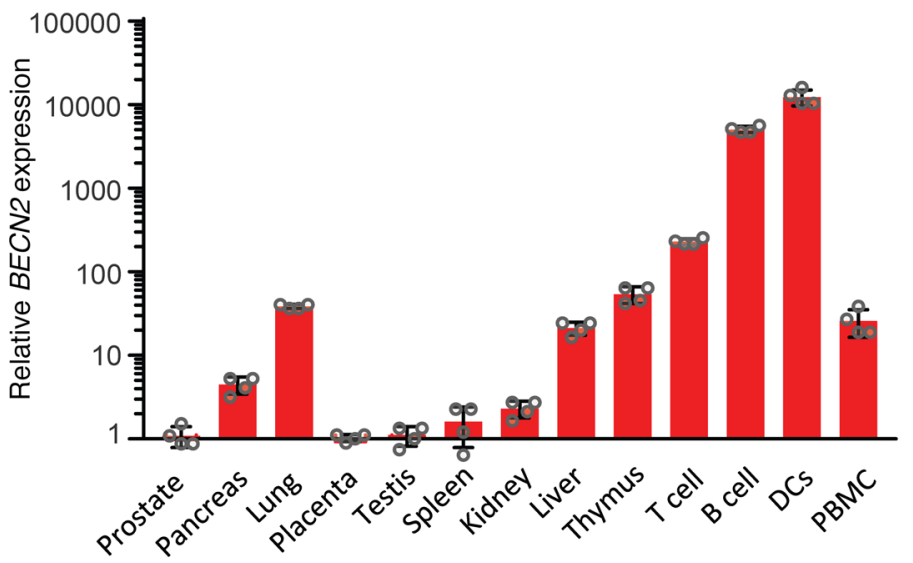

B

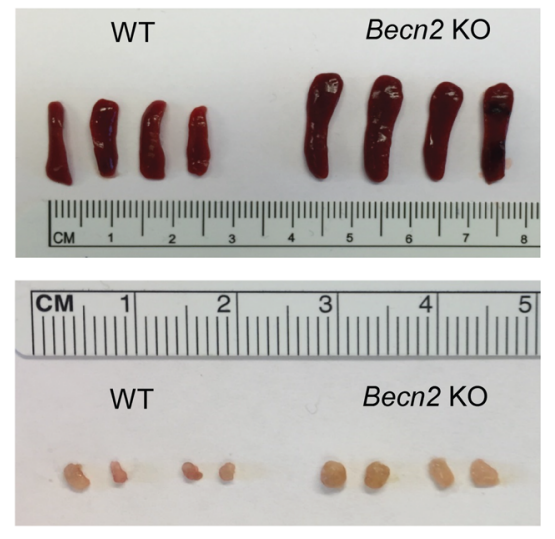

C

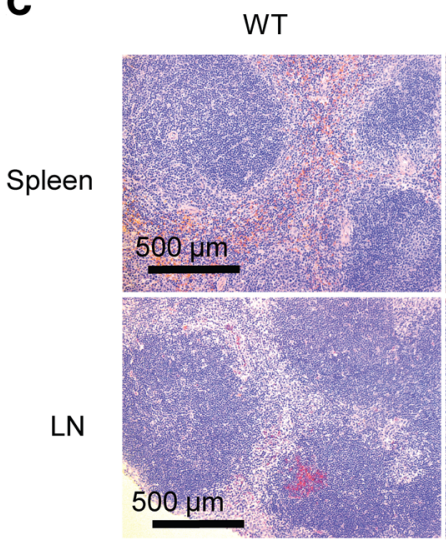

Becn2 KO

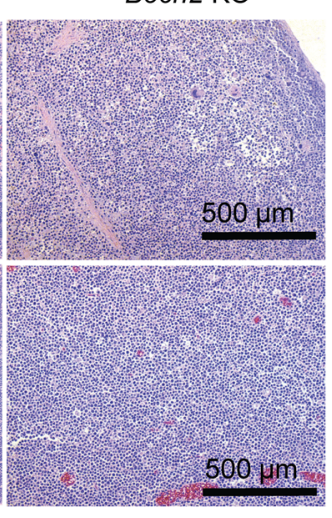

D
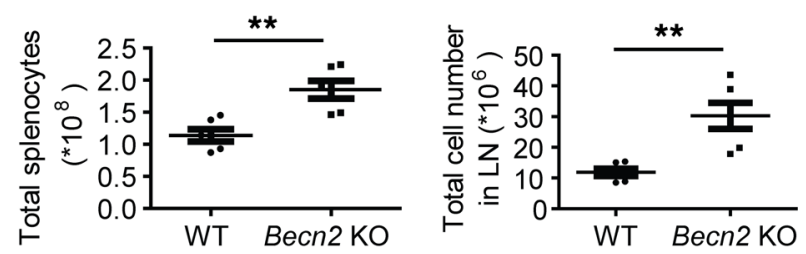

E
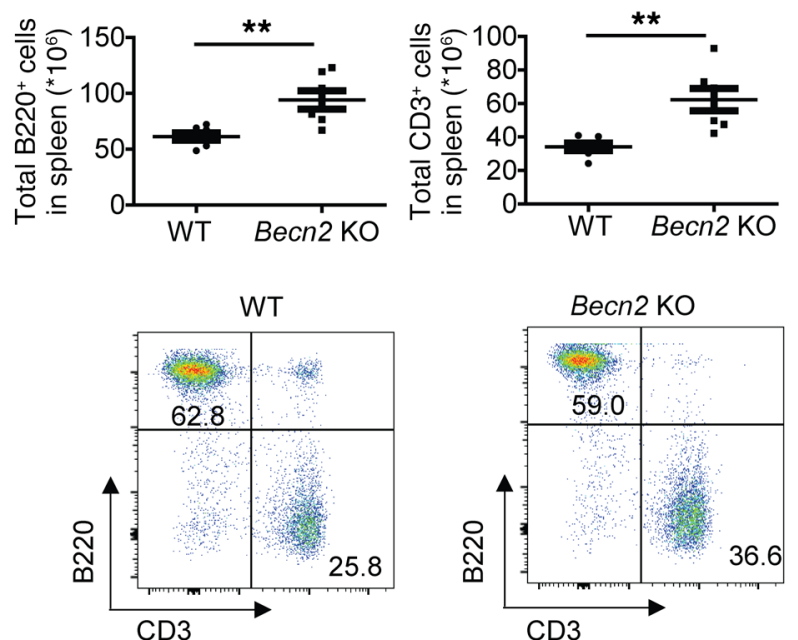

$\mathbf{F}$
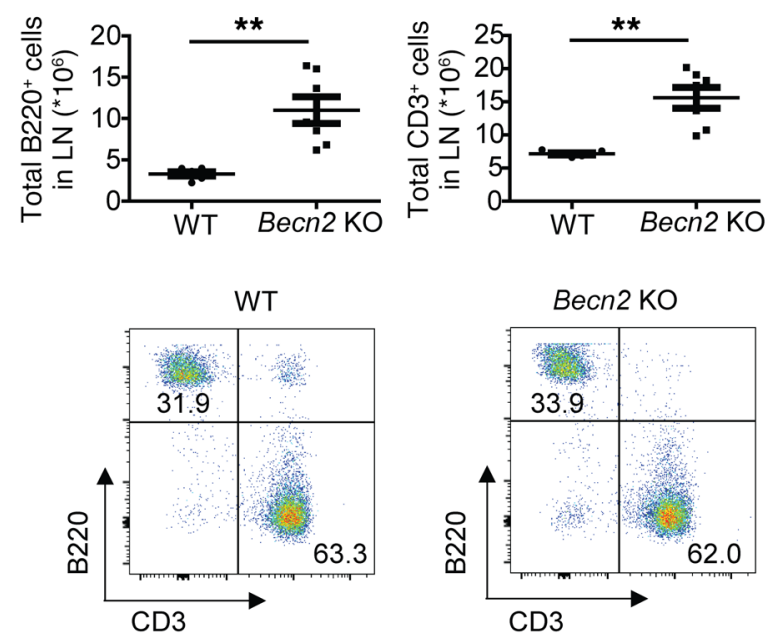

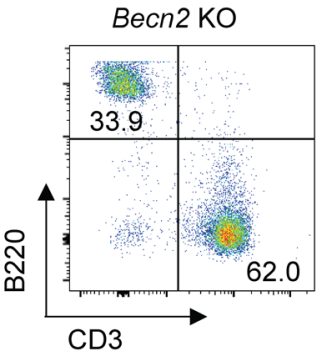

Figure 1. Splenomegaly and lymphadenopathy in Becn2-deficient mice. (A) Real-time PCR (RT-PCR) analysis of human Beclin 2 expression in different tissues and cells. The mRNA levels of BECN2 in different tissues or cells were normalized to the mRNA levels of the GAPDH gene. Data are plotted as mean \pm SEM and are representative of at least 3 independent experiments. (B) Sizes of spleens (upper) and lymph nodes (LNs, lower) from Becn2-KO and WT mice ( $n=4$ per group). (C) H\&E staining of spleen and lymph nodes of Becn2-KO and WT mice. Images are representative of 4 mice in each group. (D) Total numbers of splenocytes and lymphocytes from lymph nodes counted from 6 - to 8-week-old Becn2-KO and WT mice ( $n=6$ mice per group). (E and F) Representative flow cytometry plots and quantification of 6- to 8-week-old B220+ and $C D 3^{+}$cell populations in spleens $(\mathbf{E})$ and lymph nodes $(\mathbf{F})(n=8$ mice per group). Statistical differences between groups were calculated using Student's unpaired $t$ test. ${ }^{*} P<0.01$. 
A BMDCs -WT $=$ Becn2 $\mathrm{KO}$
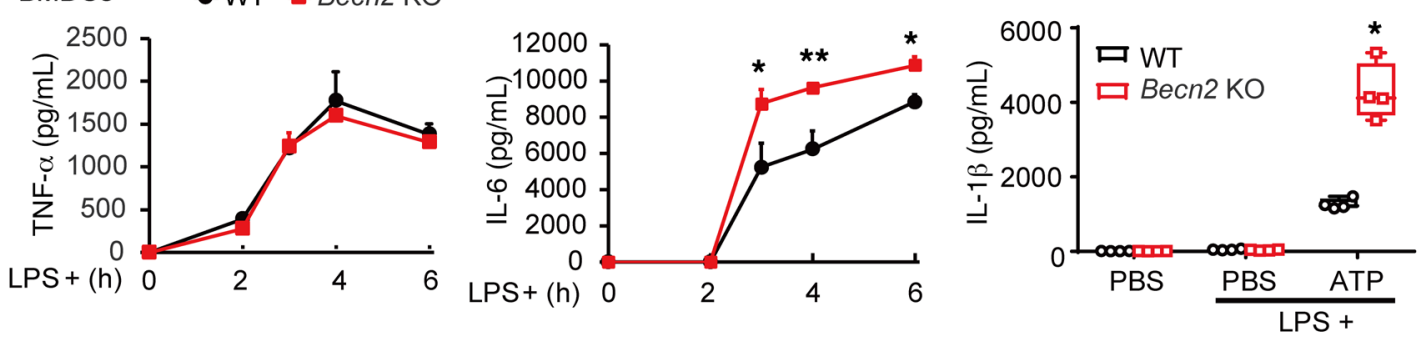

B BMDMs $-\mathrm{WT} \cdot$ Becn2 $\mathrm{KO}$
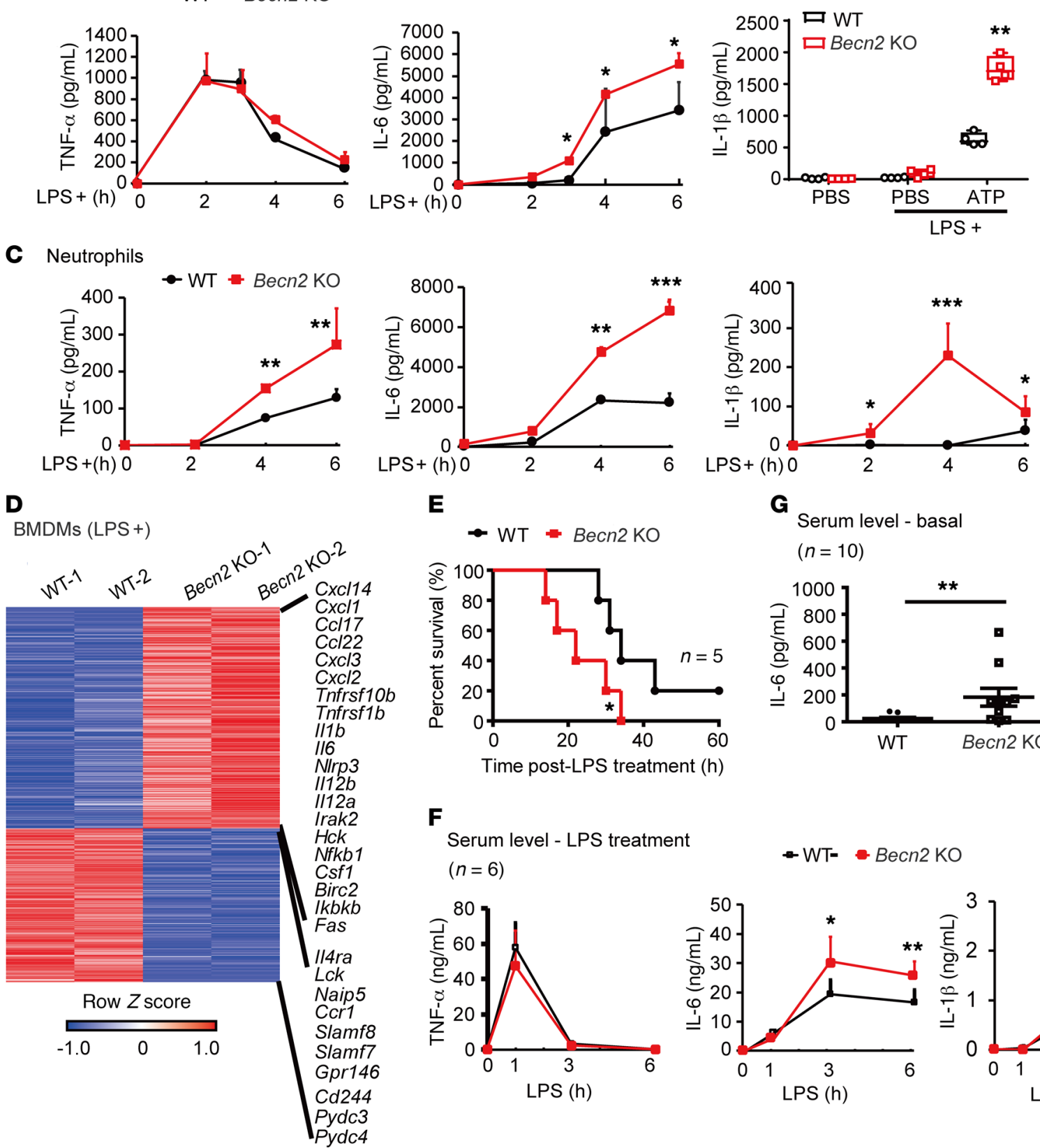

G Serum level - basal
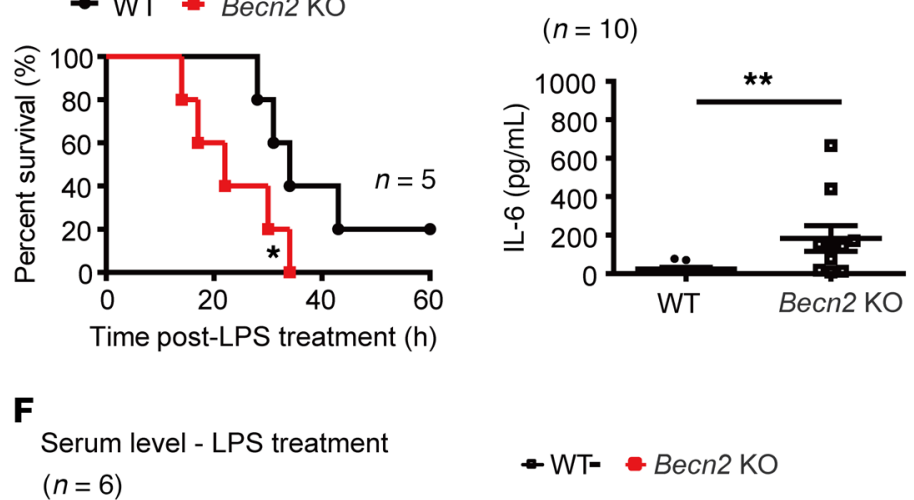

-WT- Becn2 KO
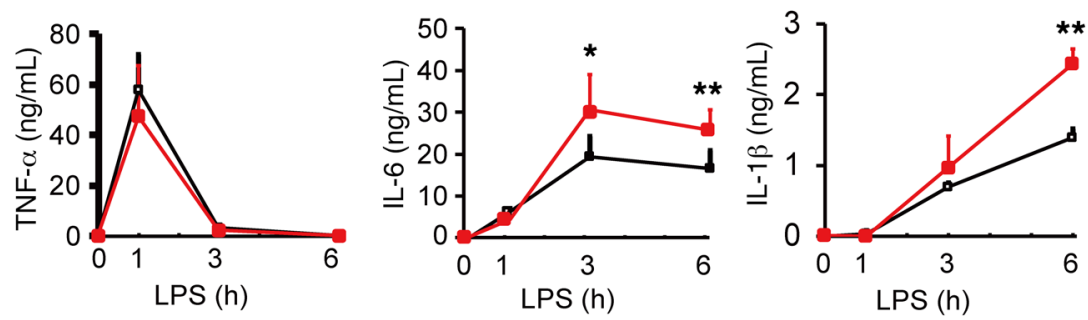

Figure 2. Ablation of Becn2 increases proinflammatory cytokine production and mouse sensitivity to LPS-induced septic shock. (A-C) Production of TNF- $\alpha$, IL-6, and IL-1 $1 \beta$ by BMDCs (A), BMDMs (B), and peritoneal neutrophils (C) treated with LPS (100 ng/mL) alone or followed by ATP (5 mM) treatment for 1 hour. (D) Heatmap of the differentially expressed genes (DEGs) in Becn2-KO BMDMs compared with WT BMDMs after 3 hours of LPS (100 ng/mL) stimulation. The most significant differentially expressed genes in the TNF signaling, NOD-like receptor signaling, cytokine-cytokine receptor interaction, and chemokine-signaling pathways between Becn2-KO BMDMs and WT BMDMs are listed on the right side. (E) Survival of 6- to 8- week-old WT and Becn2-KO mice after highdose LPS (i.p. $30 \mathrm{mg} / \mathrm{kg}$ ) treatment ( $n=5$ mice per group). (F) Cytokine levels in serum samples from 6- to 8- week-old WT and Becn2-KO mice after high-dose LPS (i.p. $30 \mathrm{mg} / \mathrm{kg}$ ) treatment ( $n=6$ mice per group). (C) IL-6 levels in 8- to 12-week-old mouse sera ( $n=10$ mice per group). Data (A-C) are plotted as mean \pm SEM and are representative of at least 3 independent experiments ( $n=2$ mice each time/group). Statistical differences between groups were calculated using Student's unpaired $t$ test $(\mathbf{A}-\mathbf{C}, \mathbf{F}$, and $\mathbf{G})$ and log-rank test $(\mathbf{E}) .{ }^{*} P<0.05 ;{ }^{* *} P<0.01 ;{ }^{* * *} P<0.001$. 
A
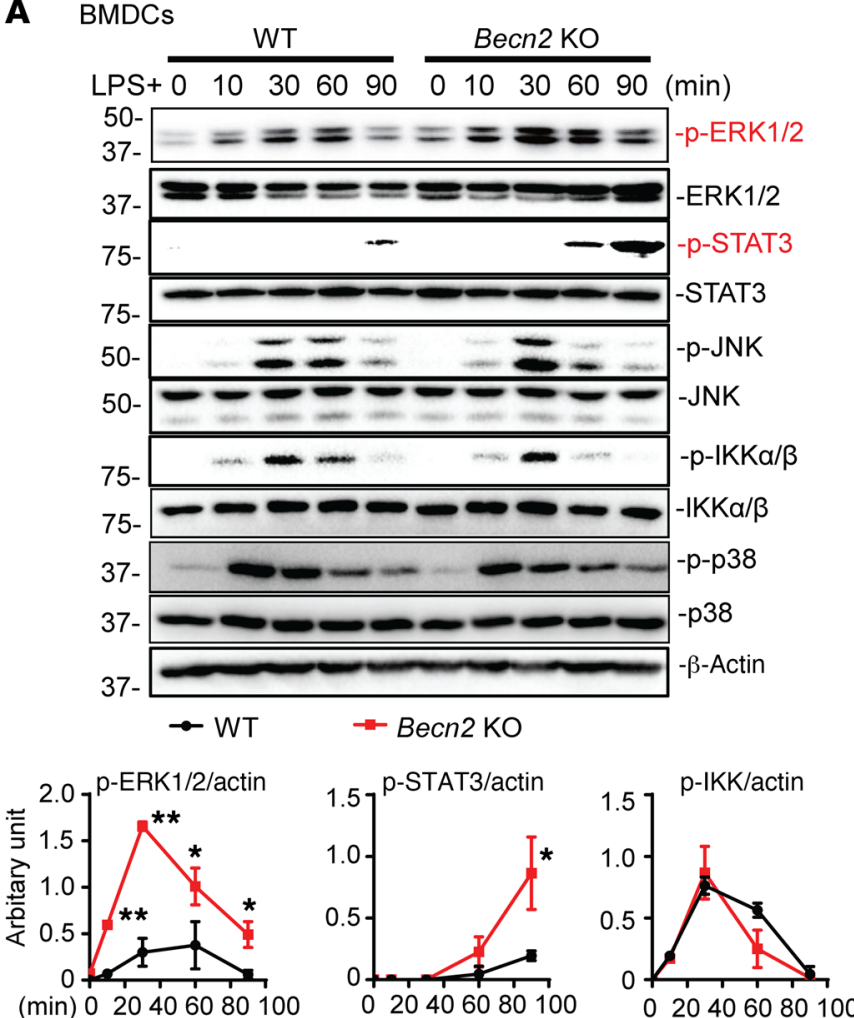

020406080100
B

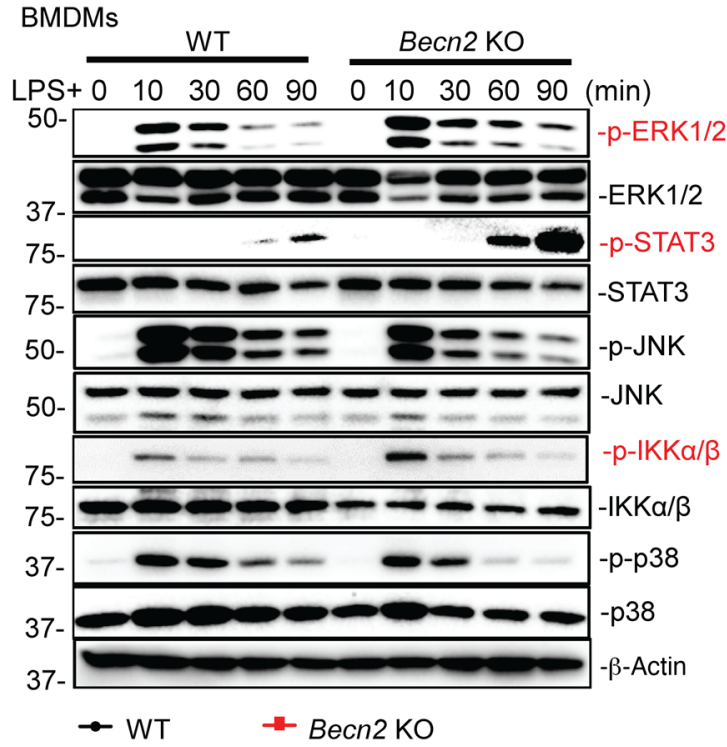

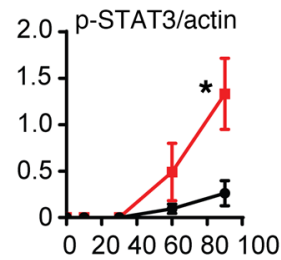

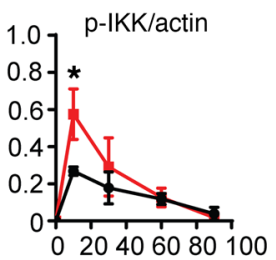

C

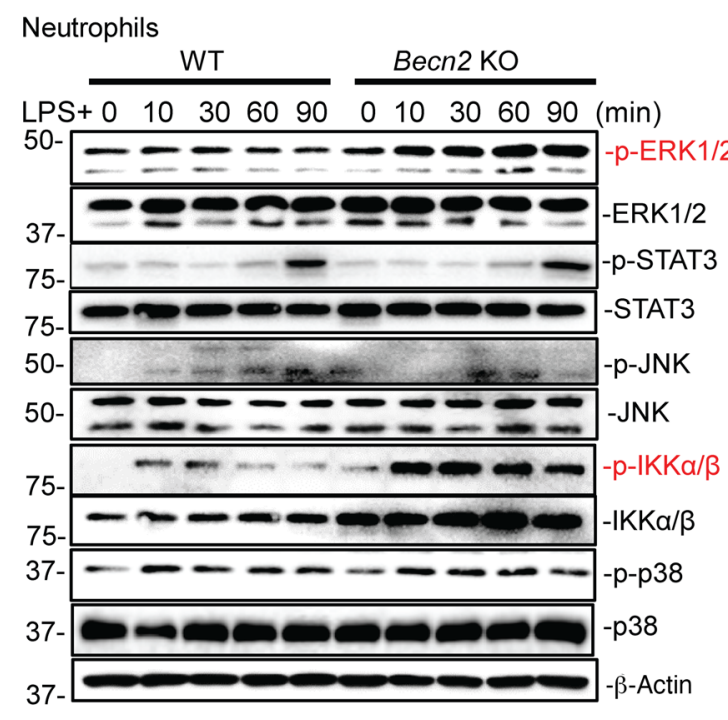

$\rightarrow$ WT

$\rightarrow$ Becn2 KO

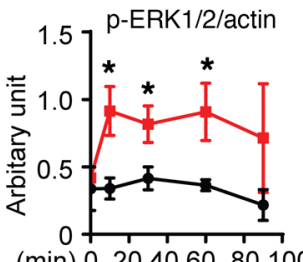

(min) 020406080100

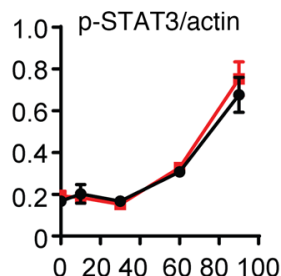

020406080100

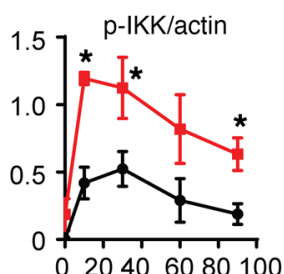

Figure 3. Becn2 deficiency enhances ERK, NF-KB, and STAT3 signaling. (A-C) Immunoblot analysis of cell lysates from WT or Becn2-KO BMDCs (A), BMDMs (B), or peritoneal neutrophils (C) treated with LPS (100 ng/ $\mathrm{mL}$ ) for the indicated time points using the indicated antibodies. Samples were run on parallel gels contemporaneously $(\mathbf{A}-\mathbf{C})$. Bottom panels: quantitative comparison of activation of signaling molecules between WT and Becn2-KO cells based on band intensity of 3 independent experiments. Data are plotted as mean \pm SEM. Statistical differences between groups were calculated using Student's unpaired $t$ test (A-C). ${ }^{*} P<0.05 ;{ }^{* *} P<0.01$ mice, we next tested to determine whether the NF- $\mathrm{KB}$ and MAPK signaling pathways were affected by Beclin 2 deficiency. Western blot analysis using different immune cells revealed that the phosphorylation of ERK1/2 was enhanced in Becn2-deficient DCs (Fig- ure 3A), macrophages (Figure 3B and Supplemental Figure 3A), and peritoneal neutrophils (Figure $3 \mathrm{C}$ ) compared with WT control cells after LPS or poly(I:C) treatment. The phosphorylation of STAT3 was also markedly enhanced in Becn2-deficient DCs and macro- 
A

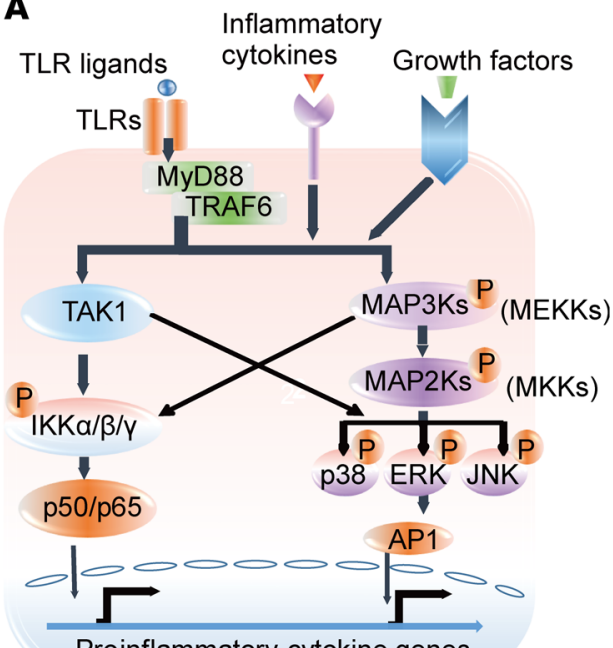

D

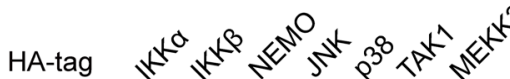

Flag-Beclin $2+++++++$

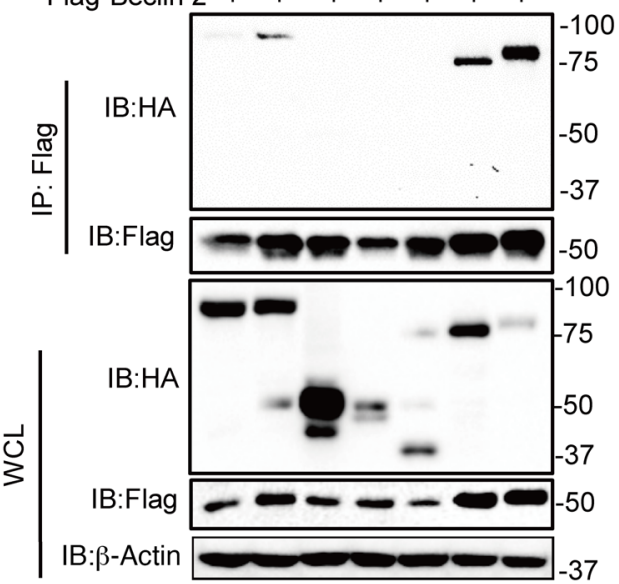

B

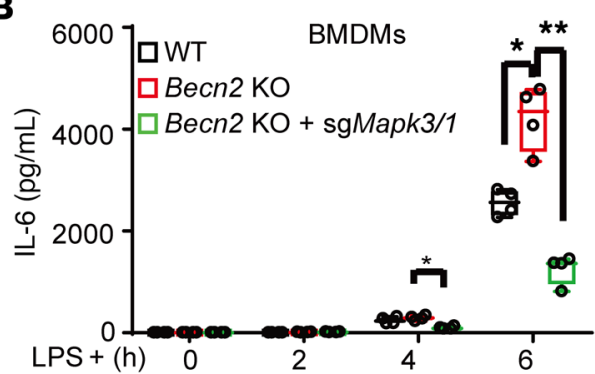

C

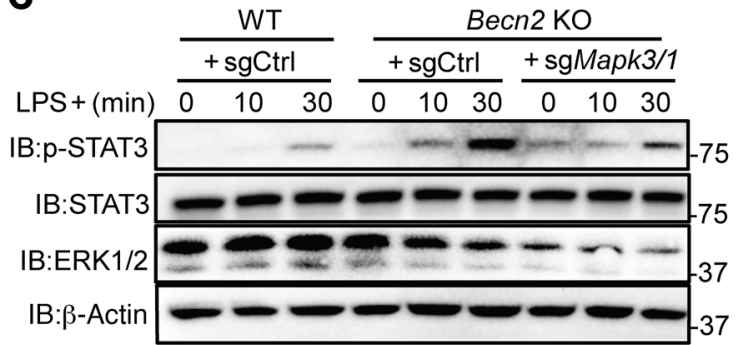

\section{G}

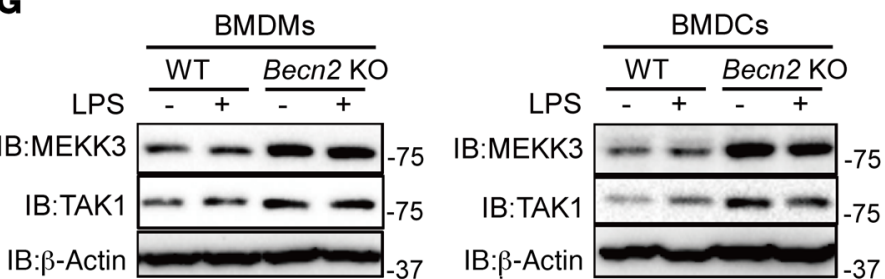

IB: $\beta$-Actin ${ }_{-37}$ IB: $\beta$-Actin

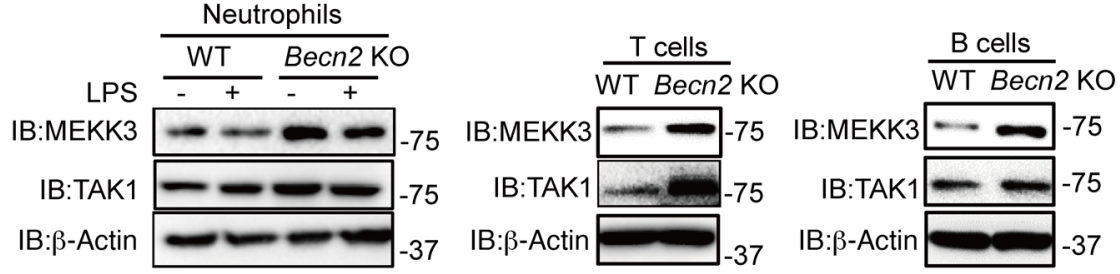

E
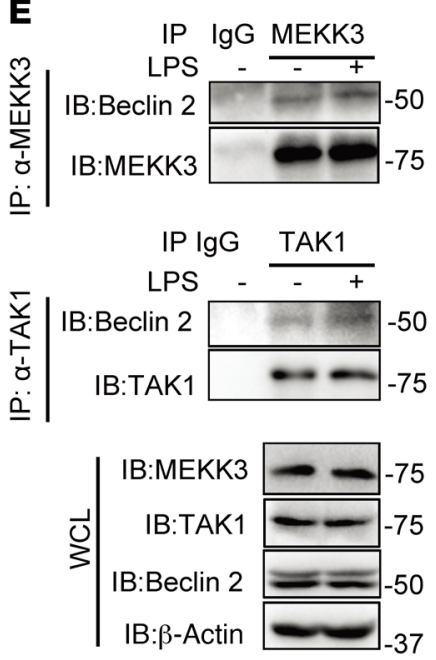

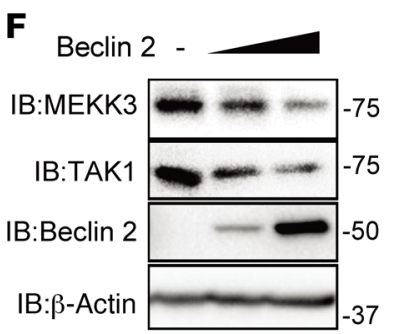

H HA-MEKK3 $\frac{\text { DMSO }}{++} \frac{\mathrm{CQ}}{++} \frac{\text { BafA1 }}{++t+\frac{3 M A}{++}} \frac{\text { MRT }}{++}$ Flag-Beclin $2-+\quad-\quad+\quad+\quad-\quad+\quad-\quad+\quad+$

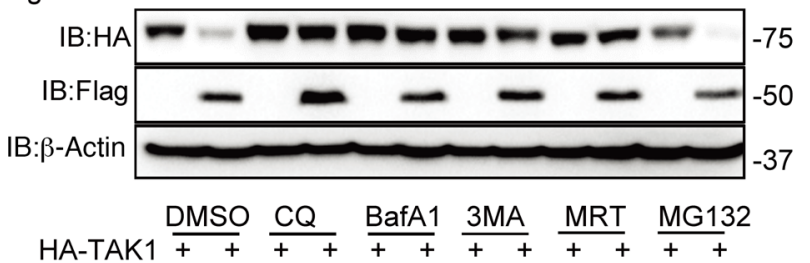
Flag-Beclin $2-+-+{ }_{-}+-+{ }_{-}+-+$

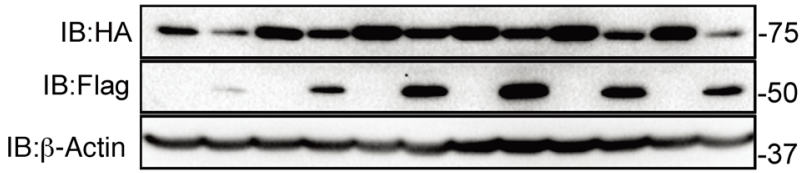


Figure 4. Beclin 2 inhibits ERK1/2 signaling by targeting MEKK3 and TAK1 for autophagic degradation. (A) Schematic illustration of proinflammatory gene expression controlled by key adaptors, kinases, and transcription factors involved in NF- $\mathrm{KB}$ and MAPK signaling. (B) IL- 6 production from WT, Becn2-KO, and sgRNA-guided Mapk3/1-KO plus Becn2-KO macrophages treated with LPS $(100 \mathrm{ng} / \mathrm{mL})$ for the indicated periods. (C) Immunoblot analysis of p-STAT3, STAT3, and ERK1/2 in WT, Becn2-KO, and sgRNAguided Mapk3/1-KO plus Becn2-KO macrophages treated with LPS (100 $\mathrm{ng} / \mathrm{mL}$ ). (D) $293 \mathrm{~T}$ cells cotransfected with the Flag-Beclin 2 plasmid alone or together with an HA-tagged plasmid were immunoprecipitated using anti-Flag beads, then immunoblotted with the indicated antibodies. (E) BMDMs were left untreated or treated by LPS $(100 \mathrm{ng} / \mathrm{mL})$ for 3 hours. Cell lysates of BMDMs were immunoprecipitated using MEKK3- or TAK1-specific antibodies against each protein, then immunoblotted with indicated antibodies. (F) Immunoblot analysis of endogenous TAK1 and MEKK 3 in the lysates of $293 \mathrm{~T}$ cells transfected with an increasing amount of pcDNA-Beclin 2 plasmid (0, 500, and $1000 \mathrm{ng} / 10^{6}$ cells). (C) Immunoblot analysis of TAK1 and MEKK3 in cell lysates of WT and Becn2-KO T cells, B cells, and macrophages, neutrophils, and DCs before and after LPS (100 $\mathrm{ng} / \mathrm{mL}, 2$ hours) treatment. Samples were run on parallel gels contemporaneously. (H) 293T cells were transfected with HA-TAK1 or HA-MEKK3 alone or together with Flag-Beclin 2 plasmid, followed by treatment for 6-10 hours with 3MA (5 mM), BafA (500 nM), CQ $(10 \mu \mathrm{M})$, MRT68921 $(1 \mu \mathrm{M})$, or MG132 $(10 \mu \mathrm{M})$. Cell lysates were immunoblotted with the indicated antibodies. Data are representative of at least 3 independent experiments. Statistical differences between groups were calculated using 1-way ANOVA with Tukey's multiple comparisons test (B). ${ }^{*} P<0.05 ;{ }^{*} P<0.01$.

phages, but not in neutrophils, after LPS or poly(I:C) treatment (Figure 3, A-C, and Supplemental Figure 3A). Notably, a moderate but significant increase in I $\mathrm{B}$ kinase (IKK) phosphorylation was observed in Becn2-deficient macrophages and neutrophils upon LPS stimulation (Figure 3, B and C). However, we did not observe any appreciable difference in ERK1/2 or STAT3 phosphorylation between Becn2-KO and WT DCs or macrophages upon CpG oligonucleotide, Pam3CSK4, or TNF- $\alpha$ treatment (Supplemental Figure 3, B-D), consistent with the cytokine production in Supplemental Figure 2C. Furthermore, we tested the signaling pathways in BECN2-KO human monocytes (THP1) upon LPS stimulation and found that BECN2-KO THP1 cells generated by the CRISPR/ Cas9 system had increased ERK1/2 and IKK signaling activities compared with WT controls (Supplemental Figure 3E). In addition, the IL-1 $\beta$ precursor (pro-IL-1 $\beta$ ) levels in BECN2-KO THP1 cells and BMDMs were both increased compared with those in WT cells after LPS treatment (Supplemental Figure 3, E and F).

Beclin 2 inhibits ERK1/2 signaling by targeting MEKK3 and TAK1 for autophagic degradation. The NF- $\mathrm{BB}$ and $\mathrm{p} 38$ signaling pathways have been known to control proinflammatory cytokine production (refs. 22, 23, and Figure 4A), but the role of ERK1/2 signaling in IL-6 expression is not clear. For this reason, we knocked out Mapk3/1 (the genes encoding ERK1/2) in Becn2-deficient macrophages using the CRISPR/Cas9 system. Cotransduction of Mapk3/1-specific sgRNAs and Cas9 into Becn2-deficient macrophages markedly reduced IL- 6 production compared with the transduction of control sgRNA (Figure 4B). Consistently, the phosphorylation of STAT3 in Becn2-deficient macrophages transduced with Mapk3/1-specific sgRNA was also reduced to a level similar to that in WT cells (Figure 4C). These results suggest a predominant role of ERK signaling in IL-6 production and STAT3 signaling in Becn2-deficient macrophages.
We next sought to determine how Beclin 2 negatively regulates ERK1/2 signaling. Because signaling cascades of MAPK pathways, such as the ERK1/2 signaling pathway, are known to be controlled via a 3-tiered process from MAP3Ks to MAP2Ks and then MAPKs (Figure 4A and ref. 24), we performed coimmunoprecipitation and Western blot analyses to identify the potential interacting proteins of Beclin 2. We showed that Beclin 2 strongly interacted with MEKK3 (Map3k3), but weakly interacted with IKK $\beta$ and TAK1 (Map3k7) (Figure 4D). The endogenous interactions of Beclin 2 with TAK1 and MEKK3 were also detected, but such interactions were not affected by LPS stimulation (Figure 4E). Furthermore, Beclin 2 strongly interacted with MKK1, MKK2, and MKK3, but not with ERK1, ERK2, ERK5, JNK, or p38 (Supplemental Figure 4A), suggesting that Beclin 2 regulates ERK1/2 signaling through the control of its upstream kinases. To further determine how Beclin 2 regulates TAK1- and MEKK3-mediated signaling, we tested to determine whether Beclin 2 could target TAK1 and MEKK3 for degradation. We transfected 293T cells with increasing amounts of Beclin 2 expression vector and found that the endogenous protein levels of both TAK1 and MEKK3 were markedly reduced with increasing Beclin 2 expression (Figure $4 \mathrm{~F}$ ). In contrast, we did not observe appreciable changes in HA-tagged MKK1, MKK2, or MKK3 protein levels with increasing Beclin 2 expression (Supplemental Figure 4B). Consistently, the protein levels of MEKK3 and TAK1 were markedly increased in Becn2-deficient immune cells, such as DCs, macrophages, neutrophils, and T cells, compared with WT controls (Figure 4G), while the mRNA levels of both MEKK3 and TAK1 had no appreciable differences between WT and Becn2-KO cells (Supplemental Figure 4C). We next determined the levels of phosphorylated TAK1 (p-TAK1) and p-MEKK3 in immune cells to test the possibility that loss of Beclin 2 could induce more TAK1 or MEKK3 phosphorylation for enhanced ERK signaling. Although increased p-TAK1 levels (in Beclin 2-KO BMDMs, BMDCs, and T cells) and p-MEKK3 (in BMDMs) were evident in Becn2-KO cells compared with WT cells, the total TAK1 and MEKK3 levels were also increased in these cell types (Supplemental Figure 4, D and E). In contrast, no appreciable difference in the phosphorylation of TAK1 was observed between WT and KO cells in the cell types that express comparable TAK1 levels, including neutrophils and B cells (Supplemental Figure 4D), suggesting that Beclin 2 mainly regulates the protein level of TAK1, but not its phosphorylation.

To further determine how Beclin 2 mediates the degradation of TAK1 and MEKK3, we used the autophagy inhibitors 3-methyladenine (3MA), bafilomycin A (BafA), chloroquine (CQ), or MRT68921 (ULK1/2 dual kinases inhibitor) to inhibit autophagy-dependent degradation and a proteasome inhibitor (MG132) to inhibit the proteasomal degradation pathway. We found that the Beclin 2mediated degradation of TAK1 and MEKK3 was significantly inhibited by the autophagy inhibitors, but not by MG132 (Figure 4H). Consistently, endogenous TAK1 and MEKK3 were also increased in autophagy/lysosome inhibitor-treated $293 \mathrm{~T}$ cells at the protein level, but with few changes at the mRNA level (Supplemental Figure $4, F$ and $G$ ), suggesting that the autophagic pathway is required for the degradation of TAK1 and MEKK3 proteins by Beclin 2.

Beclin 2 mediates the degradation of TAK1 and MEKK3 through an ATG9A-dependent, but ATG16L/LC3B/Beclin 1-independent, autophagic pathway. Beclin 2 is involved in autophagy and inter- 
A
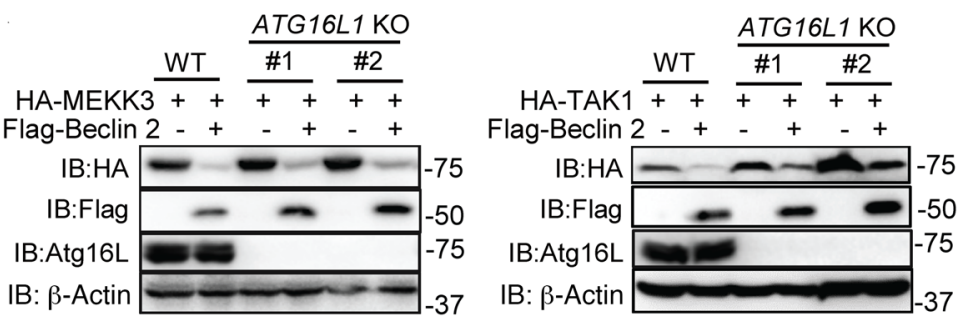

EmpVec

Flag-Beclin 2 Percent
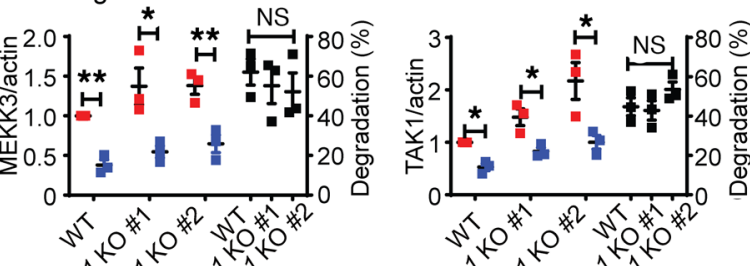

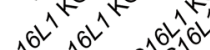

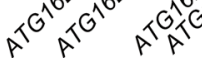

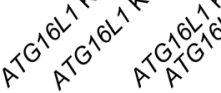

B
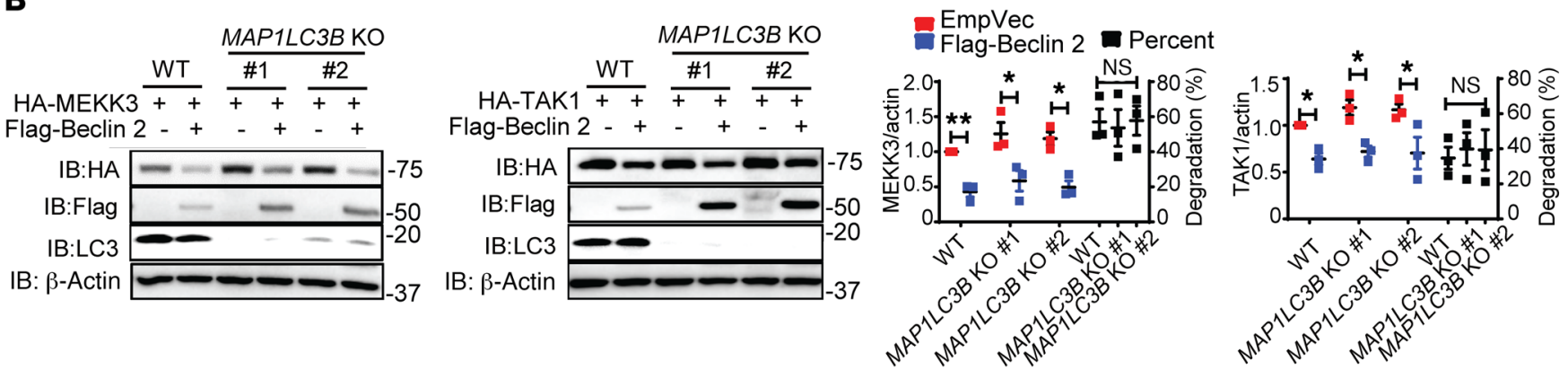

C
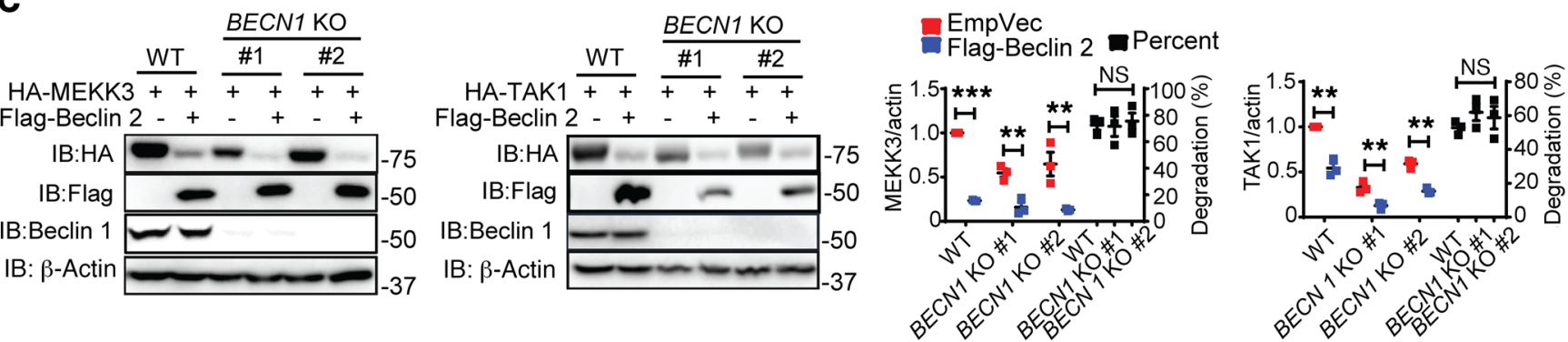

D

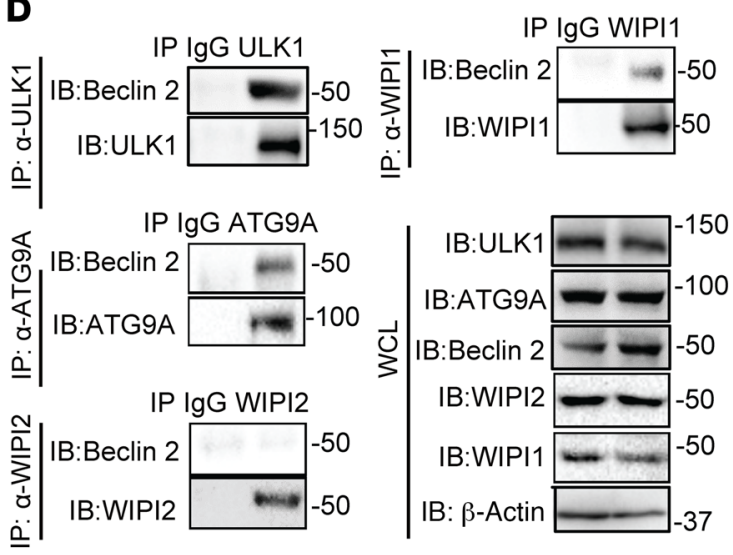

Figure 5. Beclin 2 mediates degradation of TAK1 and MEKK3 through ATC16L/LC3/ Beclin 1-independent pathway. (A-C) WT 293T cells and sgRNA-guided ATC16L-KO (A), MAP1LC3B-KO (B), or BECN1-KO (C) 293T cell clones were transfected with HA-TAK1 or HA-MEKK3 alone or together with Flag-Beclin 2 plasmid, followed by immunoblotting with the indicated antibodies. Samples shown in B were run on parallel gels contemporaneously. Right panels: quantitative analysis of HA-TAK1 and HA-MEKK3 expression and degradation percentages in WT and $\mathrm{KO}$ cells after normalization based on band intensity of 3 independent experiments. Data are plotted as mean \pm SEM. (D) Cell lysates of $293 \mathrm{~T}$ cells were immunoprecipitated using specific antibodies against each protein, then immunoblotted using indicated antibodies. Statistical differences between EmpVec-transfected and Flag-Beclin 2-transfected cells were calculated using Student's unpaired $t$ test (A-C). Statistical differences of degradation percentages between WT and KO groups were calculated using 1-way ANOVA with Dunnett's multiple comparisons test $(\mathbf{A}-\mathbf{C}) .{ }^{*} P<0.05 ;{ }^{*} P<0.01 ;{ }^{* *} P<0.001$.

acts with known binding partners of Beclin 1 in the class III PI3K complex, including ATG14, VPS34, and AMBRA1 (8), but how Beclin 2 mediates degradation of target proteins through the autophagic pathway remains unknown. Macroautophagy requires the hierarchically ordered activities of ATG proteins recruited at the phagophore assembly site (PAS) to form a double-membrane autophagosome $(1,2)$. However, recent studies indicate that autophagy could occur in alternative forms that do not require the hierarchical actions of all ATG proteins to form autophagosomes, but rather a set of ATG proteins that are recruited to a preexisting double-membrane structure for autophagosome formation $(3,4$, 25). To further decipher the molecular mechanisms of Beclin 2mediated autophagic degradation of TAK1 and MEKK3, we first determined whether Beclin 2-mediated degradation could be blocked in ATG protein-deficient cells. We knocked out ATG16L and MAP1LC3B (2 genes that are essential for autophagosome membrane elongation in macroautophagy) in $293 \mathrm{~T}$ cells and found that Beclin 2-mediated TAK1 and MEKK3 degradation could not 
be blocked in the cells deficient in either ATG16L or LC3B (Figure 5 , A and B). Ablation of BECN1 (a gene essential for nucleation in macroautophagy) also failed to block the degradation of TAK1 and MEKK3 (Figure 5C). Quantitative analysis of TAK1 and MEKK3 protein levels and degradation efficiency in WT and KO cells also indicated that Beclin 2-mediated TAK1 and MEKK3 degradation were not compromised in ATG16L/MAP1LC3B/BECN1-deficient cells compared with WT cells (Figure $5, A^{-} C$ ), suggesting that ATG16L, LC3B, and Beclin 1 are not essentially required for Beclin 2-mediated degradation of TAK1 and MEKK3.

We next sought to determine the key autophagic proteins that are essential for Beclin 2-mediated degradation of TAK1 and MEKK3. Coimmunoprecipitation of Beclin 2 and its potential binding partners involved in autophagy machinery revealed that Beclin 2 interacted with WIPI1, WIPI2, ATG9A, and ULK1 (Supplemental Figure 5, A and B). We also confirmed the endogenous interactions of Beclin 2 with ULK1, ATG9A, and WIPI1, but not with WIPI2 (Figure 5D). We next generated ATG9A-KO, ULK1-KO, and WIPI1/2-KO cells using CRISPR/Cas9 technology and found that ATG9A ablation completely abolished Beclin 2mediated TAK1 and MEKK3 degradation, while KO of ULK1 partially blocked degradation (Figure 6, A and B). However, WIPI1 or WIPI2 deficiency had little or no effect on Beclin 2-mediated MEKK3 degradation (Supplemental Figure 5C). We further compared the endogenous protein levels of MEKK3 and TAK1 in different $A T G-K O$ cells as well as in ATG9A:ATG16L-double-KO (ATG9A:ATG16L-DKO) cells. Although the protein levels of MEKK3 and TAK1 in MAP1LC3B-KO, ATG16L1-KO, and BECN1KO cells were slightly increased (Supplemental Figure 5D), much higher levels of MEKK3 and TAK1 were observed in ATG9AKO and ULK1-KO cells. Furthermore, these 2 protein levels in ATG9A:ATG16L-DKO cells were similar to those in ATG9A singleKO cells, but much higher than those in ATG16L single-KO cells (Supplemental Figure 5D). These data further demonstrate that ATG9A and ULK1, but not ATG16L/LC3/Beclin 1, play a dominant role in the degradation of MEKK3 and TAK1.

Beclin 2 promotes the fusion of MEKK3-associated $A T G 9 A^{+}$vesicles with phagophores by interacting with STX 5 and STX6 for MEKK3 degradation. ATG9A is the only transmembrane ATG protein, and the ATG9A-associated vesicle is essential for the membrane assembling in autophagosome formation (26-29). The recruitment of ATG9A vesicle to phagophores requires the activation of the ULK1 complex, including ULK1 and ATG13 (26-28). Indeed, we showed that Beclin 2-mediated MEKK3 degradation was markedly impaired in ATG13 shRNA-knockdown (shRNA-KD) cells compared with WT cells (Supplemental Figure 6A). However, further experiments showed that ATG9A interacted with ULK1 and MEKK3, but not with ATG13 or FIP200 (Supplemental Figure 6B). To further understand the role of ATG9A and ULK1 in Beclin 2-mediated MEKK3 degradation, we tested to determine whether ATG9A and ULK1 were required for the interaction between Beclin 2 and MEKK3. Coimmunoprecipitation and immunoblot analysis revealed that Beclin 2 almost completely lost the ability to interact with MEKK3 in ATG9A-KO and ULK1-KO cells (Figure $6, C$ and D). Beclin 2 also failed to be recruited to $\mathrm{ATG} \mathrm{A}^{+}$vesicles in ULK1-KO cells (Supplemental Figure 6C). These results suggest that ATG9A-ULK1 is the key to bridging the interaction between Beclin 2 and MEKK3. To further demonstrate their intracellular interactions, we performed confocal microscopic analysis and found that the colocalization of GFP-MEKK3 and Beclin 2 was significantly impaired in ATG9A-KO and ULK1-KO cells compared with WT cells (Figure 6E). Furthermore, GFP-MEKK3 was translocated into lysosome in Flag-Beclin 2-overexpressed WT cells, but such translocation was significantly compromised in ATG9A- or ULK1-deficient cells, as shown by Pearson's coefficient analysis (Figure 6F). These data demonstrate the critical role of ULK1 and ATG9A in Beclin 2-mediated degradation of TAK1 and MEKK3 through the engagement of ATG9A as a bridge for Beclin 2-ATG9A-MEKK3 complex formation. To confirm the ATG9A engagement in MEKK3 degradation in primary myeloid cells and to determine whether the degradation requires LPS stimulation, we determined the endogenous interaction between MEKK3 and ATG9A in BMDMs and found that MEKK3 could bind to ATG9A regardless of LPS stimulation (Supplemental Figure 6D). Furthermore, no appreciable change in MEKK3 levels was detected before or after LPS stimulation in WT, Becn1-KO, or Becn2-KO BMDMs (Supplemental Figure 6E), suggesting that Beclin 2-mediated ATG9A-dependent autophagic degradation of MEKK3 is independent of LPS-induced TLR4 signaling.

To further dissect the molecular mechanisms by which Beclin 2 mediates MEKK3 degradation through $\mathrm{ATG}^{+} \mathrm{A}^{+}$vesicles, we reasoned that Beclin 2 might promote the membrane fusion of ATG9A vesicles with phagophore to form autophagosome. Indeed, ectopic expression of Beclin 2 in 293T cells increased the vesicle fusion with phagophores for autophagosome formation, as shown in the transmission electron microscopy (TEM) images (Supplemental Figure 7, $A$ and $B)$. Therefore, we next sought to determine the potential interaction between Beclin 2 and a series of RAB GTPases and SNARE proteins for their involvement in membrane fusion (30-32). Coimmunoprecipitation and Western blot analyses showed that Beclin 2 could interact with RAB7A, RAB8A, RAB32, VAMP8, syntaxin 5 (STX5), STX6, STX7, and STX8 (Supplemental Figure 7C). We next generated $\mathrm{KO}$ cells of each Beclin 2-interacting partner using CRISPR/Cas9 technology and tested to determine whether specific gene KO would partially or completely block the MEKK3 degradation. We found that ablation of either STX 5 or STX 6 could partially block the Beclin 2-mediated MEKK3 degradation (Figure 7A), while $\mathrm{KO}$ of other genes had little or no effect (Supplemental Figure 7D). Importantly, Beclin 2-mediated MEKK3 degradation could be completely blocked in STX5:STX6-DKO cells (Figure 7A). To further investigate the physiological role of STX5 and STX6 in Beclin 2-mediated MEKK3 degradation, we first demonstrated the endogenous interactions of Beclin 2 with STX5 and STX6 (Figure 7B). Next, we examined the translocation of MEKK3 in WT and STX5:STX6-DKO cells using TEM analysis (by APEX2-enabled staining) and found that MEKK3 could be associated with single-membrane vesicles or transported to autophagosomes (double-membrane structures) in WT cells. In contrast, MEKK3 was barely detected in autophagosomes in STX5:STX6-DKO cells, but accumulated with single-membrane vesicles (Figure $7 \mathrm{C}$ ). In line with the TEM observation, we performed membrane fractionation to enrich autophagosomes and immune-isolated Flag-tagged ATG9A ${ }^{+}$vesicles, respectively, from cells of different genotypes. We found significant amounts of MEKK3 in ATG9A vesicles among all genotypes 
A

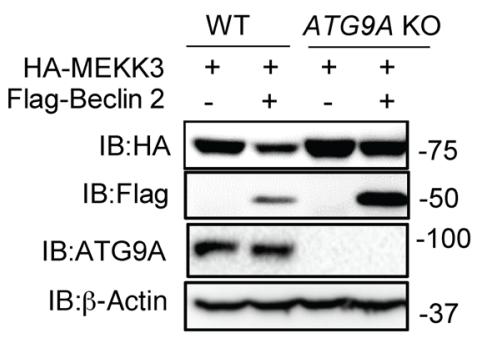

\section{EmpVec}

Flag-Beclin 2 Percent

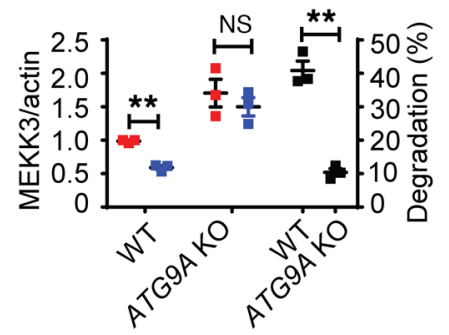

C
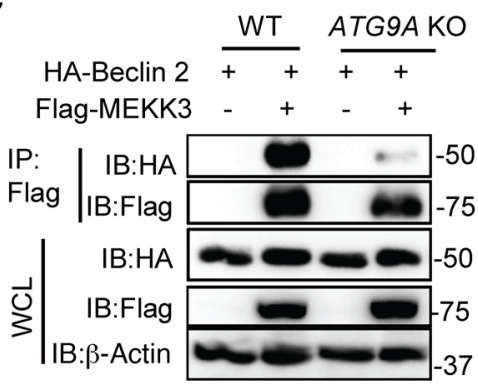

D

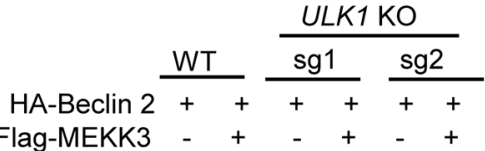

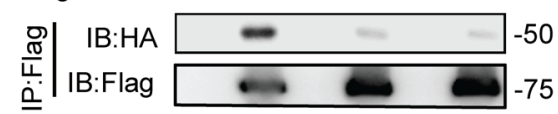

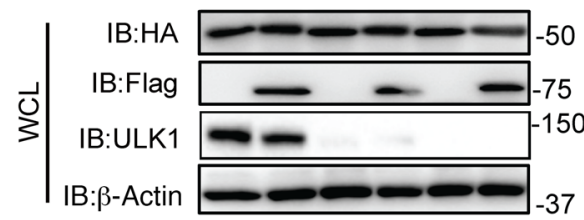

HA-TAK1 $\frac{\text { WT }}{+++\frac{A T G 9 A K O}{++}}$ Flag-Beclin 2

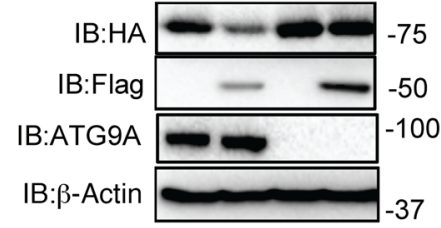

- EmpVec

Flag-Beclin 2 Percent
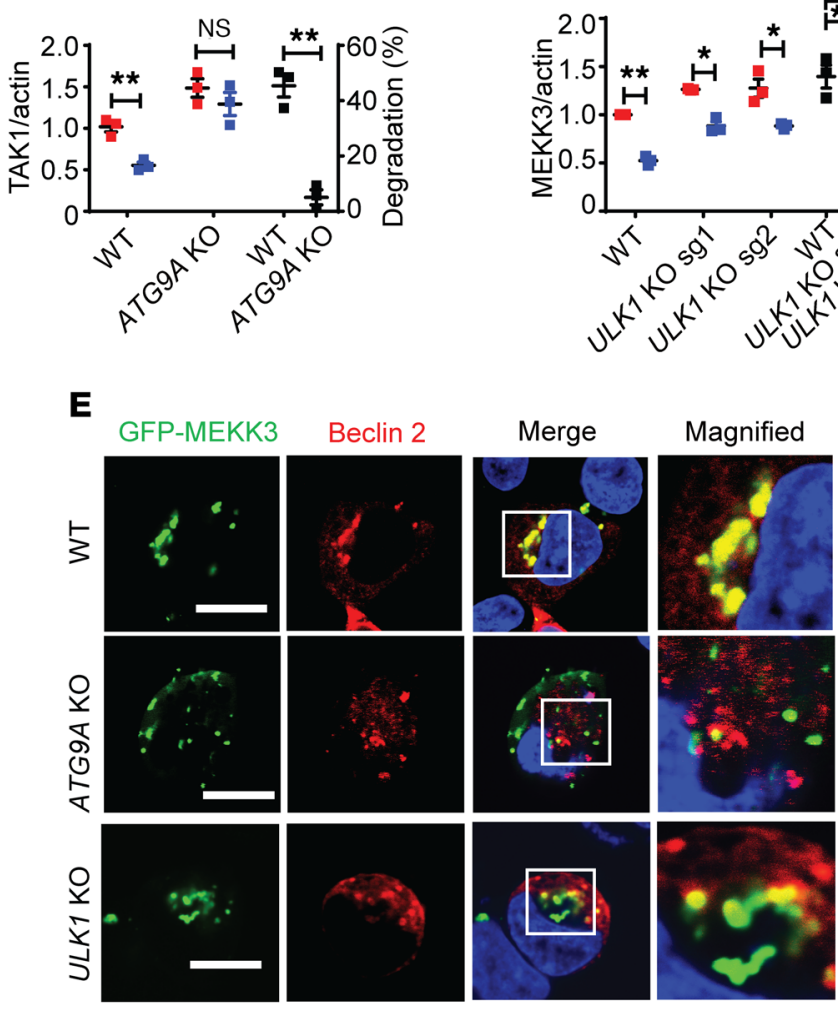

E

F

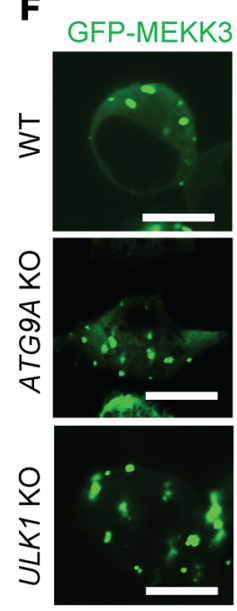

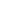

Lysotracker
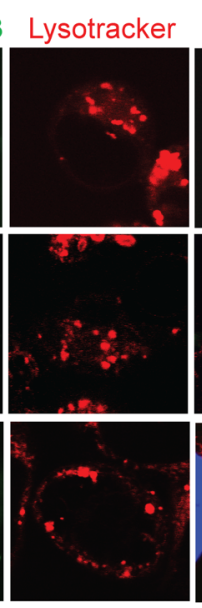

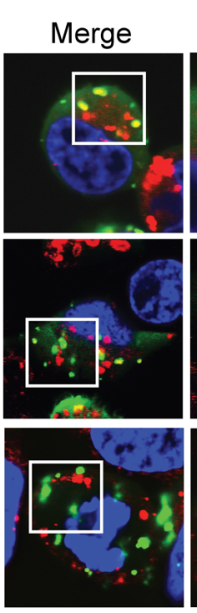

B
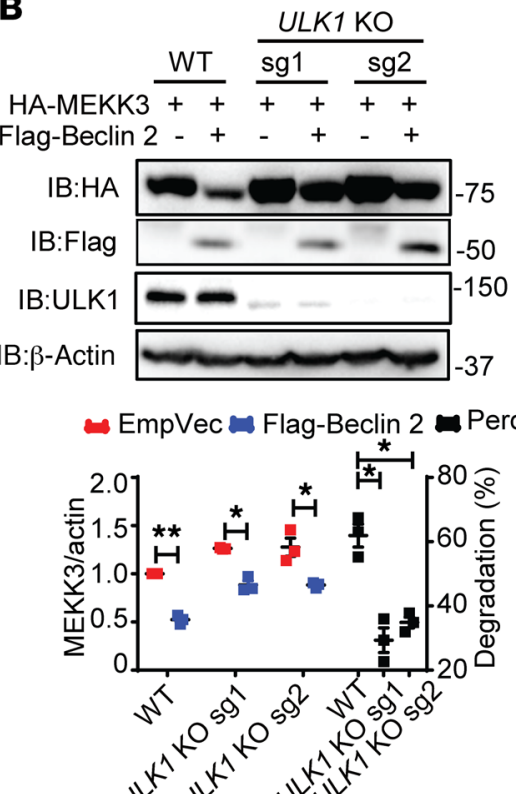

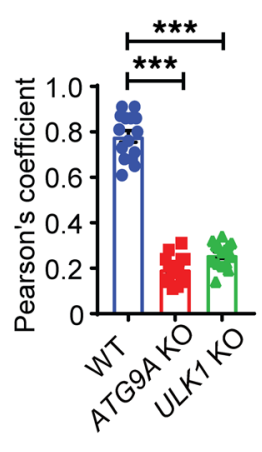

Magnified
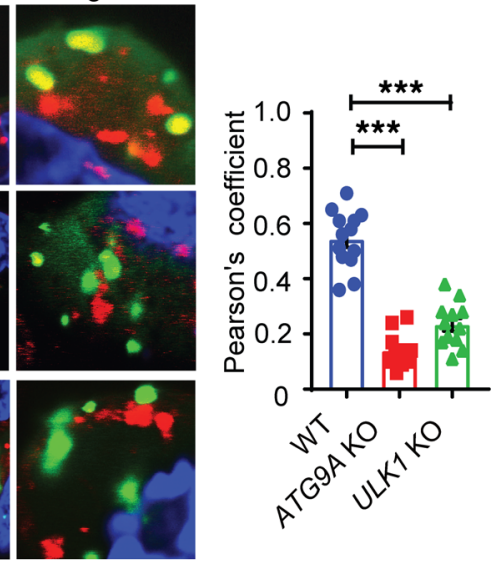
Figure 6. Beclin 2 mediates the degradation of TAK1 and MEKK3 through an ATG9A- and ULK-dependent autophagic pathway. (A and B) WT 293T cells, sgRNA-guided ATC9A-KO 293T cells (A), and sgRNA-guided ULK1KO 293T cells (B) were transfected with HA-TAK1 or HA-MEKK3 alone or together with Flag-Beclin 2 plasmid, followed by immunoblotting with the indicated antibodies. Blots were run contemporaneously with the same protein samples (A). Bottom panels: quantitative analysis of HA-TAK1 and HA-MEKK3 expression and degradation percentages in WT and KO cells after normalization based on band intensity of 3 independent experiments. (C and D) WT and ATC9A-KO (C) or ULK1-KO (D) 293T cells were transfected with the HA-Beclin 2 plasmid alone or together with FlagMEKK3. Cell lysates were immunoprecipitated using anti-Flag beads, followed by immunoblotting with the indicated antibodies. Data are representative of 3 independent experiments. (E) Confocal images of WT, ATC9A-KO, and ULK1-KO 293T cells cotransfected with GFP-MEKK3 and Flag-Beclin 2, then stained with anti-Flag antibody, followed by Alexa Fluor 633-conjugated secondary antibody staining. Hoechst 33342 was applied for nucleus staining. Scale bars: $10 \mu \mathrm{m}$. (F) Confocal images of WT, ATC9AKO, and ULK1-KO 293T cells transfected with GFP-MEKK3, then stained with lysotracker and Hoechst 33342 . Scale bars: $10 \mu \mathrm{m}$. Original magnification, $\times 1000$ (merge); $\times 2500$ (magnified). Data are plotted as mean \pm SEM. Pearson's correlation coefficient for colocalization shown in $\mathbf{E}$ and $\mathbf{F}$ was used with Image J Coloc 2 (at least 30 cells were analyzed per condition)।. Statistical differences between EmpVec-transfected and Flag-Beclin 2transfected cells were calculated using Student's unpaired $t$ test (A and B). Statistical differences between WT and KO groups were calculated using Student's unpaired $t$ test (A) or 1-way ANOVA with Dunnett's multiple comparison tests (B, E, and F). ${ }^{*} P<0.05 ;{ }^{* *} P<0.01$; ${ }^{* *} P<0.001$.

(Figure 7D). Moreover, the increased amounts of MEKK3 could be detected in $\mathrm{ATG} \mathrm{A}^{+}$vesicle fractions from BECN2-KO, STX5-KO, and STX6-KO cells compared with WT counterparts, in which much less MEKK3 was detected (Figure 7D). However, the enrichment of MEKK3 in autophagosomes could only be detected in WT cells, but not in BECN2-KO, STX5-KO, or STX6-KO cells, suggesting that the translocation of MEKK3 from $\mathrm{ATG} \mathrm{A}^{+}$vesicles to autophagosomes is markedly blocked in these KO cells (Figure 7D). Consistently, the translocation of MEKK3 into the lysosomes was also diminished in STX5:STX6-DKO 293T cells even with Beclin 2 overexpression (Figure $7 \mathrm{E})$. Taken together, these results suggest that the interaction between Beclin 2 and STX5/STX6 promotes the membrane fusion of $\mathrm{ATG} \mathrm{A}^{+}$vesicles with phagophores to form autophagosomes for MEKK3 degradation (Supplemental Figure 7E).

Ablation of Map3k3 rescues phenotypes observed in Becn2-KO mice. To further explore the physiological function of Beclin 2mediated MEKK3 and TAK1 degradation, we determined whether specific ablation of MEKK3 or TAK1 could rescue the phenotypes observed in Becn2-deficient mice. We crossed Becn2-KO mice with myeloid-specific TAK1-deleted (Map3k $7^{\Delta \mathrm{M} / \Delta \mathrm{M})}$ mice or myeloid-specific MEKK3-deleted (Map3k3 $\left.3^{\Delta \mathrm{M} / \Delta \mathrm{M}}\right)$ mice and found that myeloid-specific ablation of MEKK3 (Map3k3 $\left.3^{\Delta \mathrm{M} / \Delta \mathrm{M}}: B e c n 2-\mathrm{KO}\right)$ completely rescued the phenotypes (splenomegaly and lymphadenopathy) observed in Becn2-deficient mice (Figure 8A), whereas TAK1 deficiency (Map3k $7^{\Delta \mathrm{M} / \Delta \mathrm{M}}:$ Becn2-KO) only partially rescued the phenotypes. Consistently, histological examination revealed that $M a p 3 k 3^{\Delta \mathrm{M} / \Delta \mathrm{M}}: B e c n 2-\mathrm{KO}$ mice restored the disorganized spleen architectures observed in Becn2-deficient mice to a level similar to that of WT mice, while Map3k $7^{\Delta \mathrm{M} / \Delta \mathrm{M}}:$ Becn2-KO mice only partially restored the germinal center and nodular architecture (Figure $8 \mathrm{~B}$ ). Furthermore, the total numbers of splenocytes and $\mathrm{B}_{22} 2 \mathrm{O}^{+}$and $\mathrm{CD}^{+}$lymphocytes as well as the serum cytokine production in $M a p 3 k 3^{\Delta \mathrm{M} / \Delta \mathrm{M}}: B e c n 2-\mathrm{KO}$ mice under the untreated (basal) and LPS-induced conditions were either partially or completely restored to levels comparable to those of WT mice (Figure $8, \mathrm{C}-\mathrm{E})$. These results were further supported by a marked reduction of IL- 6 in macrophages isolated from Map3k3 $3^{\Delta \mathrm{M} / \Delta \mathrm{M}}:$ Becn2-KO and $M a p 3 k 7^{\Delta \mathrm{M} / \Delta \mathrm{M}}: B e c n 2-\mathrm{KO}$ mice compared with Becn2-KO cells (Figure $8 \mathrm{~F}$ ). Consistently with cytokine production, ablation of Map3k3 or Map3k7 in Becn2-KO macrophages markedly reduced the phosphorylation of ERK1/2 and IKK $\alpha / \beta$ to levels similar to or even lower than those in WT macrophages (Figure $8 \mathrm{G}$ ). These results suggest that myeloid-specific TAK1 and MEKK3 are critical in mediating IL-6 production, but that MEKK3 plays a predominant role in the development of splenomegaly and lymphadenopathy in Becn2-KO mice.

Increased incidence of metastatic lymphoma development in Becn2-KO mice. Based on our findings that Becn2-KO mice produce large amounts of proinflammatory cytokines such as IL-6, we reasoned that these $\mathrm{KO}$ mice may have a higher risk of developing cancer. Indeed, we observed tumor development in homozygous Becn2-KO mice at the age of approximately 20 to 32 weeks (Figure 9A). Among 38 Becn2-KO mice (from 6 to 36 weeks old), 5 (13.2\%) developed spontaneous tumors compared with no tumor development in WT mice, as expected. To determine whether myeloid-specific ablation of Map $3 k 3$ could also rescue the spontaneous tumor development in Becn2-KO mice, we checked 18 Becn2-KO:Map $3 k 3^{\Delta \mathrm{M} / \Delta \mathrm{M}}$ mice at matched ages and found that none of these mice developed tumors (Figure 9A). These results suggest that excess MEKK3-mediated signaling in Becn2-KO mice plays a critical role in spontaneous tumor development. The tumors found in Becn2-KO mice were near the neck and collarbone region (Figure 9B) and had different pathological architectures. The sizes of the livers, inguinal lymph nodes, and spleens from tumor-bearing Becn2-KO mice were dramatically increased compared with those from WT mice (Figure 9C). Based on the H\&E staining of tissues from tumor-bearing Becn2-KO mice, we also detected lung metastases in mice harboring tumor no. 1 and tumor no. 2 and liver metastases in the mouse harboring tumor no. 1 (Figure 9D). We further performed immunostaining on primary tumors and metastatic tissues to determine the tumor types in Becn2-KO mice. Immunohistochemical staining of CD3, B220, and Ki67 showed that the primary tumor and lung metastases from mouse no. 1 could be characterized as T cell lymphoma (Figure 10A), while the staining of tumor no. 2 showed a mixed population of $\mathrm{T}$ cells and $\mathrm{B}$ cells (Figure 10B). To further define the lymphoma type of tumor no. 2, we used confocal microscopy to identify the colocalization of Ki67 with either CD3 or B220 and identified it as B cell lymphoma based on the colocalization of Ki67 and B220 (Figure 10C). Among 5 lymphomas developed in Becn2-KO mice, one was T cell lymphoma and 4 were B cell lymphomas. These results suggest that Beclin 2 deficiency promotes lymphoma development with metastasis in the lung and liver.

Enhanced STAT3 activation and cytokine/chemokine expression in lymphomas of Becn2-KO mice. Since the loss of Beclin 2 leads to increased MEKK3 protein levels and ERK signaling for IL-6 production, we reasoned that the elevated IL- 6 production and persistent STAT3 activation in T and B lymphocytes in Becn2-KO 
A

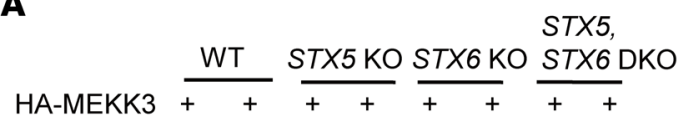

Flag-Beclin 2 - $\quad+\quad-\quad+\quad-\quad+\quad+\quad+$
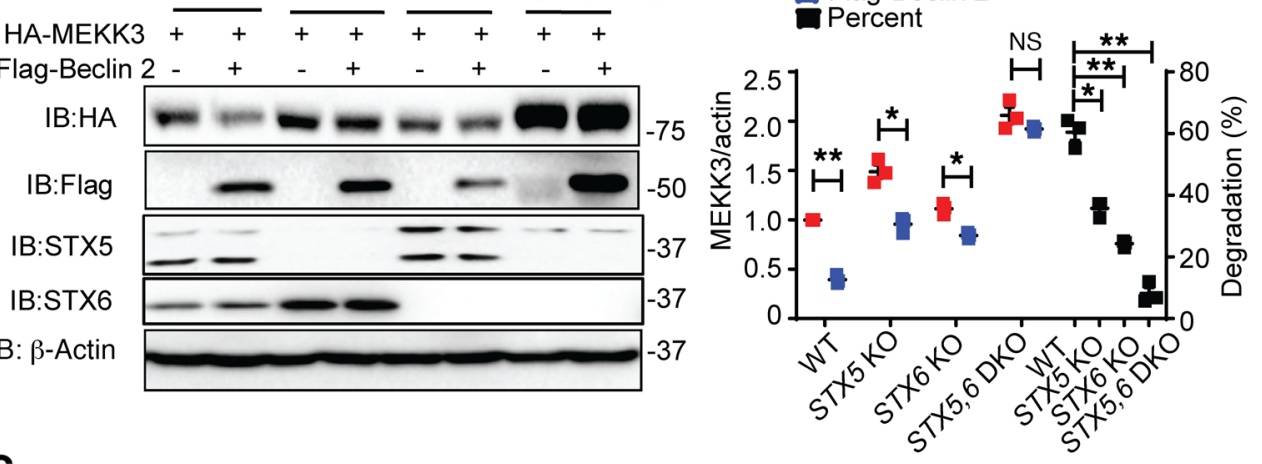

C

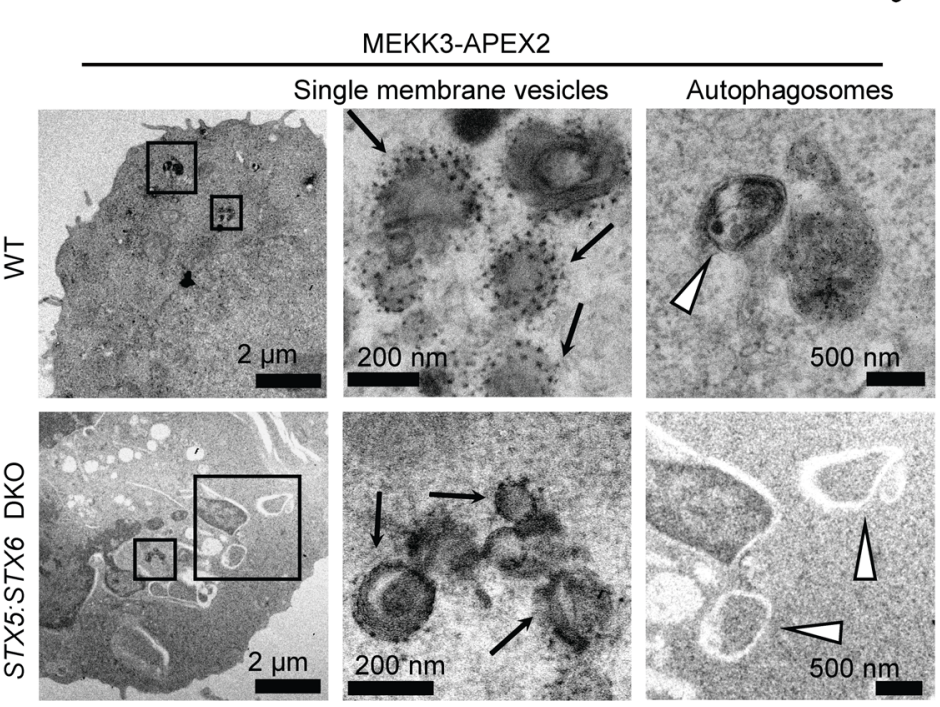

E
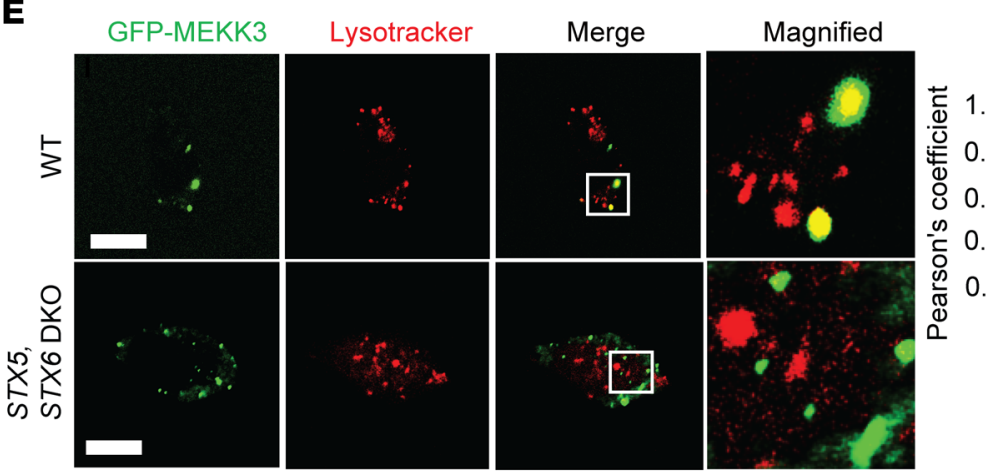

EmpVec

Flag-Beclin 2

Percent

B
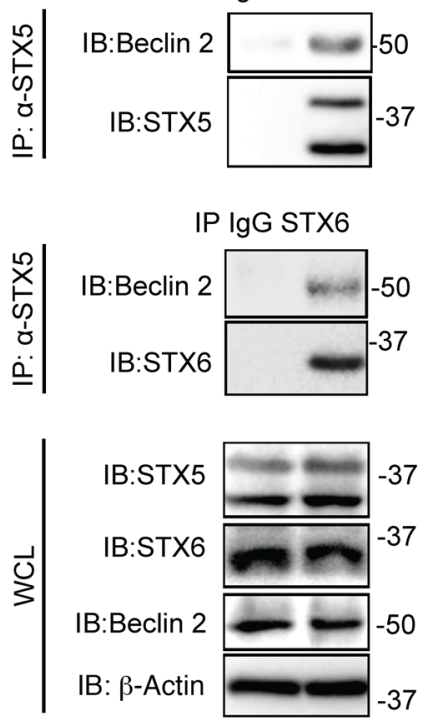

D

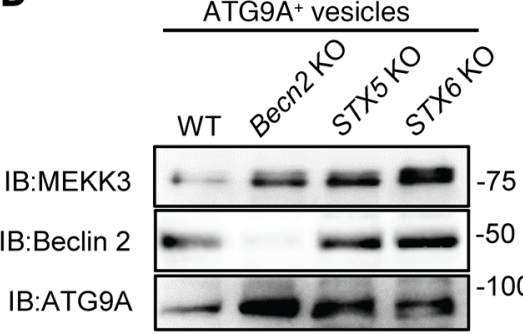

Autophagosomes

IB:MEKK3

IB:LC3-I

LC3-II

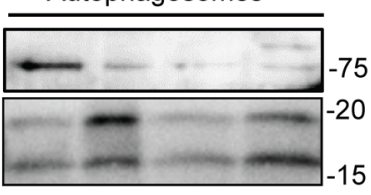

Cell lysates

IB:LC3-I

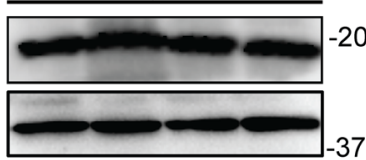

Figure 7. Beclin 2 binds to STX5/6 to promote the fusion of ATG9A vesicles with phagophores for MEKK3 degradation. (A) WT, sgRNA-guided STX5KO, STX6-KO, or STX5:STX6-DKO 293T cells were transfected with HA-MEKK3 alone or together with Flag-Beclin 2 plasmid, followed by immunoblotting with the indicated antibodies. The blots were run contemporaneously with the same protein samples. Right panel: quantitative analysis of HA-MEKK3 expression and degradation percentage. (B) Cell lysates of 293T cells were immunoprecipitated using anti-STX5 or anti-STX6 antibody, respectively, and then immunoblotted with the indicated antibodies. WCL, whole cell lysates (C) Representative TEM images of WT and STX5:STX6 DKO 293T cells cooverexpressing Flag-Beclin 2 and MEKK3-APEX2 processed in parallel under identical conditions. Black arrows indicate MEKK3-associated vesicles; white arrowheads indicate MEKK3-containing autophagosome. (D) WT, STX5-KO, and STX6-KO cells were cotransfected with Flag-ATC9A and HA-Beclin 2, while BECN2 KO 293T cells were transfected with Flag-ATC9A alone. Half of the cells were left untreated for immunoisolation of Flag-ATC9A ${ }^{+}$vesicles; the other half were pretreated by CQ ( $10 \mu \mathrm{M}, 4$ hours) to inhibit the fusion of autophagosomes with lysosomes for the enrichment of autophagosomes. Cells were resuspended in specific fractionation buffers for isolation of Flag-ATC9A+ vesicles or autophagosome enrichment, respectively, followed by immunoblotting with indicated antibodies. (E) Confocal images of WT and STX5:STX6-DKO 293T cells transfected with GFP-MEKK3 and Flag-Beclin 2, then stained with Iysotracker. Scale bars: $10 \mu \mathrm{m}$. Pearson's correlation coefficient for colocalization was used with Image J Coloc 2. Graph represents mean \pm SEM (at least 30 cells were analyzed per condition). Data are representative of 3 independent experiments. Statistical differences between EmpVec-transfected and Flag-Beclin 2-transfected cells were calculated using Student's unpaired $t$ test (A). Statistical differences of degradation percentages between WT and KO groups were calculated using 1-way ANOVA with Dunnett's multiple comparison test (A). Statistical analyses of colocalization between groups were calculated using Student's unpaired $t$ test $(\mathbf{E})$. ${ }^{*} P<0.05 ;{ }^{* *} P<0.01 ;{ }^{* *} P<0.001$. 
mice might play a critical role in tumorigenesis, as previously suggested $(11,33,34)$. Therefore, we first determined whether IL-6 directly affected $\mathrm{T}$ or B cells for the promotion of lymphoma development in Becn2-KO mice. We checked the NF- $\mathrm{B}$ and MAPK signaling in splenic T cells and B cells from Becn2-deficient mice that were potentially affected by elevated basal IL- 6 and found that both ERK1/2 and STAT3 signaling were persistently activated in Becn2-deficient $\mathrm{T}$ cells and B cells (Supplemental Figure 8, A and B). Furthermore, RNA-Seq analysis showed that many genes involved in immune responses to infectious diseases, transcriptional misregulation in cancer, NOD-like receptor and cytokine-cytokine receptor signaling, and MAPK signaling were dysregulated in Becn2-KO T cells and B cells compared with WT control cells (Supplemental Figure 8, C-F). Next, we examined STAT3 and ERK status by immunofluorescence staining in tumors or normal lymph nodes and found dramatic increases in both p-STAT3 and p-ERK levels in lymphomas from Becn2-KO mice compared with lymph nodes from WT mice (Figure 11, A and B). Immunoblot analysis further demonstrated increased STAT3 phosphorylation in Becn2-KO lymphomas and lymph nodes compared with WT control samples (Figure 11C). Notably, MEKK3 and TAK1 expression in lymphomas and lymph nodes from Becn2KO mice were consistently increased (Supplemental Figure 9A). These results suggest that STAT3 signaling is constitutively activated in Becn2-deficient lymphocytes by elevated IL- 6 production to promote lymphoma development.

We next compared gene expression profiles among WT lymph nodes, Becn2-KO lymph nodes, and Becn2-deficient lymphomas using RNA-Seq analysis. We found that many genes involved in inflammation, cell proliferation, and tumor metastasis were upregulated in Becn2-KO lymph nodes and/or lymphomas compared with WT lymph nodes (Figure 11D). In particular, the cytokine-cytokine receptor interaction, transcriptional dysregulation in cancer, cell adhesion, and cell lineage pathways were significantly altered in Becn2-KO lymphomas compared with either Becn2-KO or WT lymph nodes (Figure 11E). Furthermore, the upregulation of essential tumorigenic proinflammatory cytokines, chemokines, and oncogenes as well as the downregulation of cell-cell adhesion molecules were confirmed by real-time PCR (Figure 12A and Supplemental Figure 9B) and immunohistochemical staining (Figure 12B). In particular, Cxcr4, IL-21, Ccl3, and $\mathrm{Bcl}-7 \mathrm{a}$ were robustly expressed in tumor tissues (Figure 12, A and B). There was no appreciable difference in Bcl-2, p-Bcl-2, Bcl-xl, or Mcl-1 expression between WT and KO lymph nodes (Supplemental Figure 9, C and D), although these proteins have been reported to bind to Beclin 1 and have been implicated in promoting tumorigenesis in patients with low Beclin 1 expression $(8,35,36)$. These results suggest that the persistent activation of STAT3 signaling as well as the upregulation of Cxcr4, Ccl3, IL-21, and Bcl-7a expression may promote lymphoma development in Becn2-deficient mice. To further determine the role of IL-6 in spontaneous lymphoma development in Becn2-KO mice, we used IL-6 neutralizing antibody to treat the mice for 4 consecutive weeks and found that the levels of these upregulated key genes that were identified in Becn2-KO lymphoma samples were significantly reduced to levels similar to those of WT counterparts, including Bcl7a, Il-21, Pdcd1, Cxcr4, Tnfsf8, and Ccl3 (Figure 13A). Moreover, IL-6 neutralizing antibody treatment also significantly rescued the splenomegaly observed in Becn2-deficient mice (Figure 13B). The levels of p-STAT3 in Becn2-KO splenocytes were restored to levels similar to those of WT cells (Figure 13C). Consistently, the total numbers of splenocytes and $\mathrm{B}_{2} 2 \mathrm{O}^{+}$and $\mathrm{CD}^{+}$lymphocytes were significantly reduced compared with those in control antibody-treated Becn2-KO mice (Figure 13D). These results suggest that elevated IL-6 production is closely associated with persistent STAT3 activation and the development of splenomegaly and spontaneous lymphoma in Becn2-KO mice. Kaplan-Meier plot analysis using human cancer databases revealed that Beclin 2 expression levels were associated with overall survival rates for patients with cancer. In particular, for patients with bladder carcinoma and thyroid carcinoma, the higher levels of Beclin 2 expression were significantly associated with prolonged overall survival compared with that of patients with low Beclin 2 expression $(P<0.001)$ (Supplemental Figure 9E). Furthermore, higher expression levels of Beclin 2 were also associated with extended overall survival for ovarian cancer patients $(P<0.05)$ (Supplemental Figure 9E), but to a lesser extent. These data further suggest that Beclin 2 may have a tumor-suppressive function through innate immune regulation, STAT3 signaling, and chemokine expression.

\section{Discussion}

In this study, we showed that homozygous deletion of Becn2 led to splenomegaly, lymphadenopathy, and enhanced inflammatory responses. Elevated IL-6 production was evident in Becn2-deficient macrophages, DCs, and neutrophils upon stimulation, while TNF- $\alpha$ levels were increased in Becn2-deficient neutrophils, but not in other immune cells tested. IL-1 $\beta$ was also highly produced by Becn2-deficient immune cells compared with WT control cells due to elevated expression of pro-IL-1 $\beta$ after LPS treatment. In contrast, enhanced IL-1 $\beta$ production, but not elevated pro-IL-1 $\beta$ levels, was found in heterozygous Becn1-KO macrophages compared with WT macrophages (37). Consistent with these findings, Becn2-deficient mice were more sensitive to LPS-induced septic shock, suggesting that Beclin 2 functions as a negative regulator of the innate immune signaling pathways to control the expression of proinflammatory cytokines, such as IL- 6 and IL-1 $\beta$.

Although NF- $\kappa$ B signaling is known to drive TNF- $\alpha$ and IL-6 expression (22, 23), Becn2-deficient DCs showed increased IL-6 production, despite comparable IKK $\alpha / \beta$ activity between WT and Becn2-KO cells after LPS treatment. Further experiments showed that the ablation of ERK1/2 (Mapk3/1) by the CRISPR/Cas9 system in Becn2-deficient cells restored IL-6 production to a level similar to that in WT cells, suggesting that elevated ERK1/2 signaling is responsible for the increase in IL-6 production. Our results are consistent with the previous finding that ERK1/2 signaling antagonists inhibited IL-6 production (38). Interestingly, we found that ERK signaling only yielded differences between WT and Becn2-KO cells after LPS and poly I:C stimulation, but not CpG or Pam3CSK4 stimulation. TRIF is the adapter for TLR3/TLR4 signaling and type I IFN signaling; however, no appreciable difference in type I IFN signaling was found between WT and Becn2-KO cells, suggesting that Beclin 2 negatively regulates ERK signaling mainly through targeting the downstream kinases, such as MEKK3, but not through the TRIF signaling molecule. The downstream sig- 
A

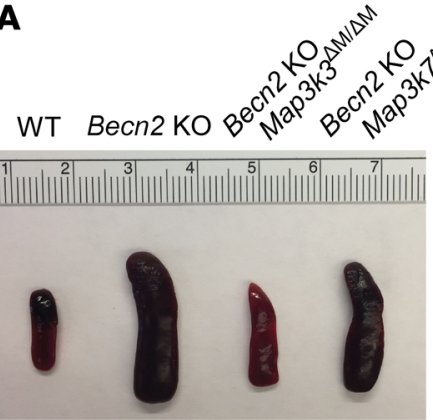

B
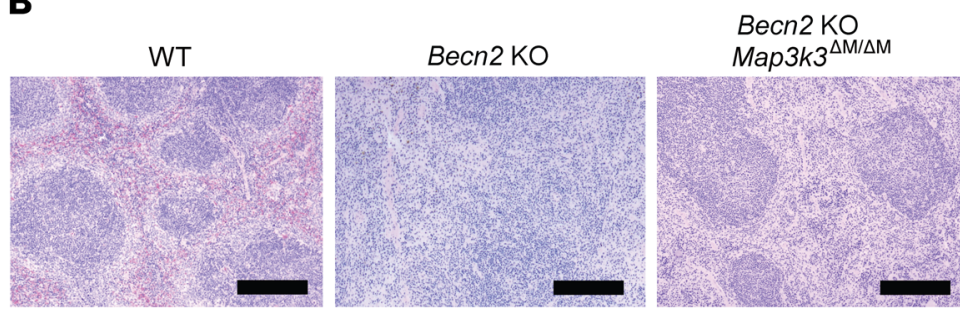

WT Becn2 KO

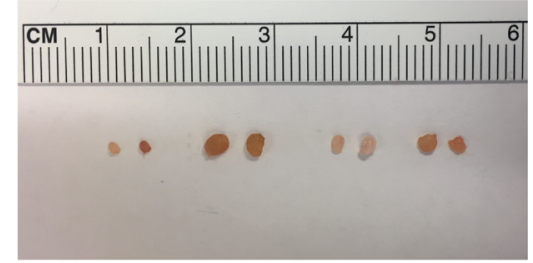

C
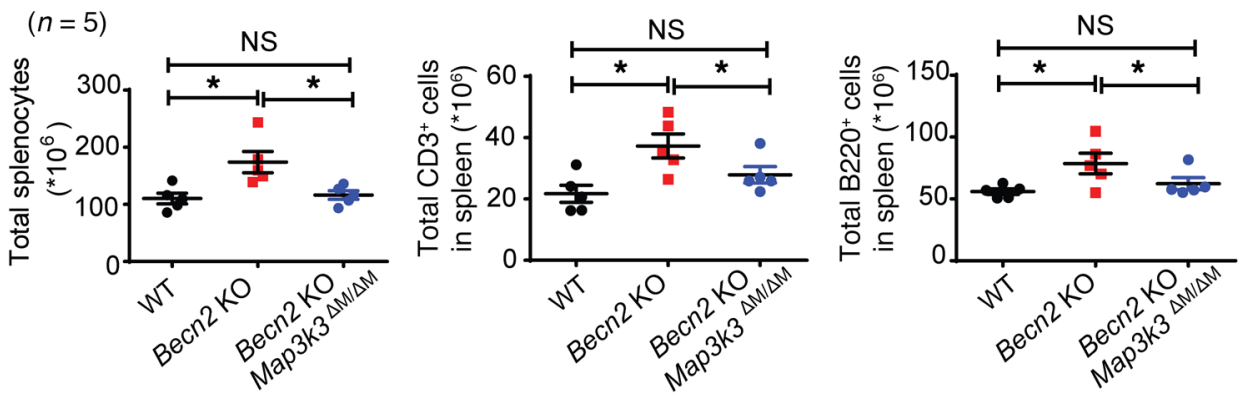

D

Serum level-basal $(n=8)$

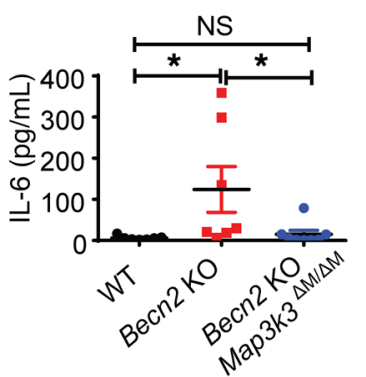

E

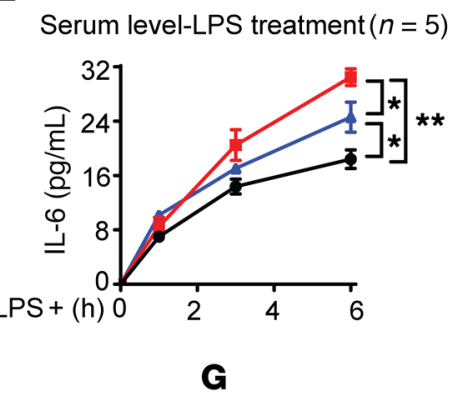

Becn2 KO $\operatorname{Map} 3 k 7^{\Delta \mathrm{M} / \Delta \mathrm{M}}$

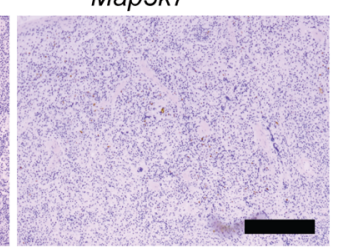

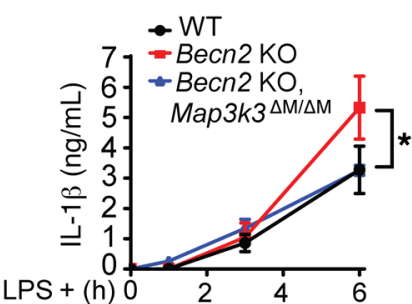

$\mathbf{F}$

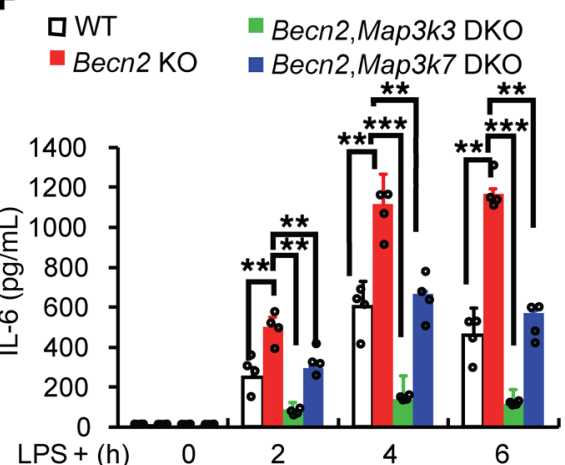

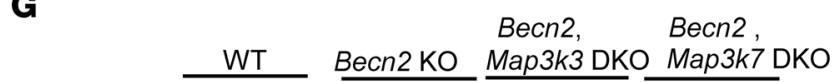

LPS + (min) $0 \begin{array}{lllllllllllll}30 & 60 & 0 & 3060 & 0 & 30 & 60 & 0 & 3060\end{array}$

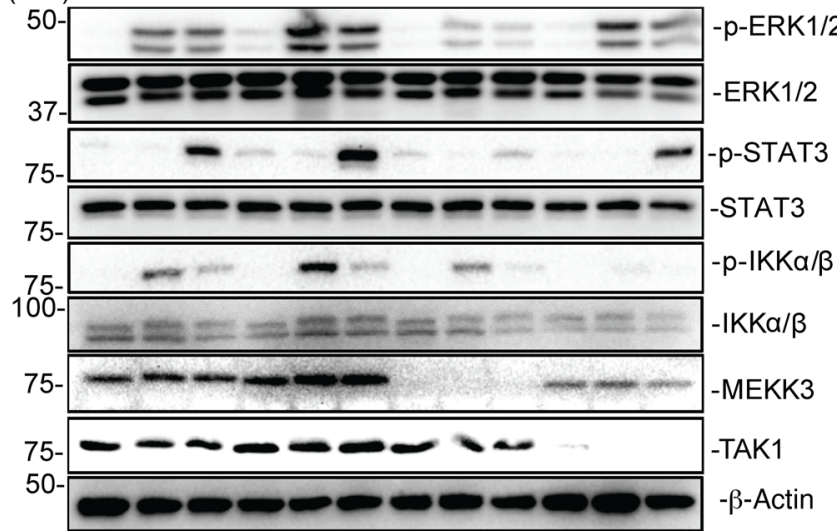

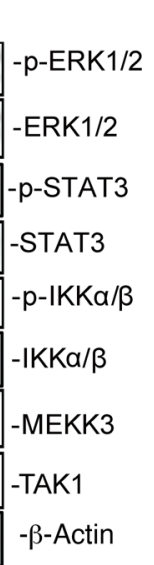

Figure 8. Ablation of Map3k3 rescues the phenotypes observed in Becn2-KO mice. (A) Spleens and lymph nodes of 6- to 8-week-old

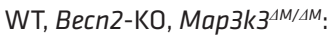
Becn2-KO, and Map3k7 $7^{\mathrm{MM} / \Delta M}$ :Becn2-KO mice. (B) H\&E staining of spleens from WT, Becn2-KO, Map3K3 ${ }^{1 \mathrm{M} / \Delta \mathrm{M}}: \mathrm{Becn} 2-\mathrm{KO}$, and Map3K $7^{\triangle M / \triangle M}: B e c n 2-K O$ mice. Scale bars: $1 \mathrm{~mm}$. Representative images ( $\mathbf{A}$ and $\mathbf{B}$ ) are shown for each group ( $n=5$ mice per group). (C) Total numbers of splenocytes and flow cytometry-based quantification of $\mathrm{B}_{22 \mathrm{O}^{+}}$and $\mathrm{CD}^{+}$cell populations in spleens counted from 6- to 8-week-old Becn2$\mathrm{KO}$ and WT mice ( $n=5$ mice per group). (D) Basal IL-6 levels in 8- to 12-week-old mouse sera ( $n$ = 8 mice per group). (E) Cytokine levels in serum samples from 6- to 8-week-old WT, Becn2-KO, and Becn2-KO:Map3k3 ${ }^{\triangle M / \Delta M}$ mice after high-dose LPS (i.p. $30 \mathrm{mg} /$ $\mathrm{kg}$ ) treatment ( $n=5$ mice per group). (F) IL-6 production by WT,

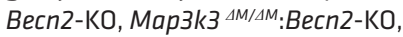
and Map3K $7^{1 \mathrm{M} / \Delta \mathrm{M}}:$ Becn2-KO macrophages treated with LPS (100 $\mathrm{ng} / \mathrm{mL}$ ) for $0,2,4$, or 6 hours (mean \pm SEM). (C) Immunoblot analysis of macrophages from WT, Becn2-KO, Map3k3 $3^{\mathrm{MM} / \Delta \mathrm{M}}: B e c n 2-K O$, and Map3K $7^{\triangle M / \triangle M}: B e c n 2-K O$ mice treated with LPS $(100 \mathrm{ng} / \mathrm{mL})$ for the indicated periods. Data ( $\mathbf{F}$ and C) are representative of 3 independent experiments. Statistical differences between groups were calculated using 1-way ANOVA with Dunnett's multiple comparisons test (C-F). ${ }^{*} P<0.05$; ${ }^{*} P<$ $0.01 ;{ }^{* * *} P<0.001$. 
A

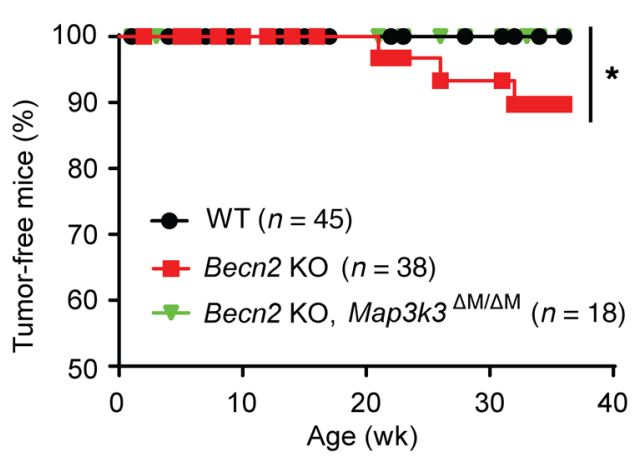

C

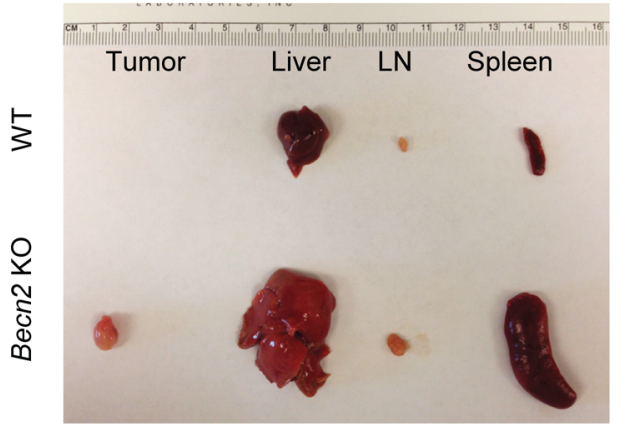

B

Becn2 KO

Tumor \#1
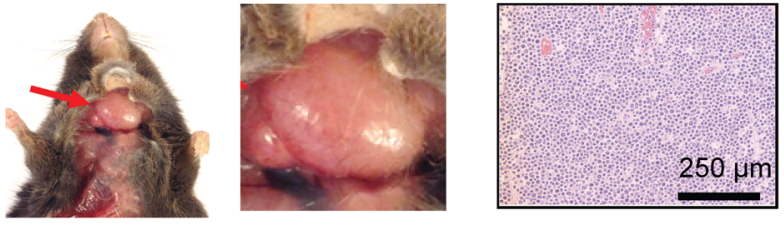

Tumor \#2
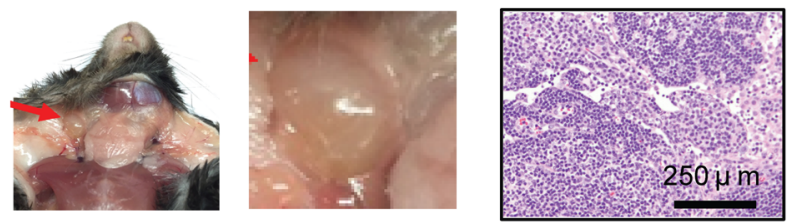

D

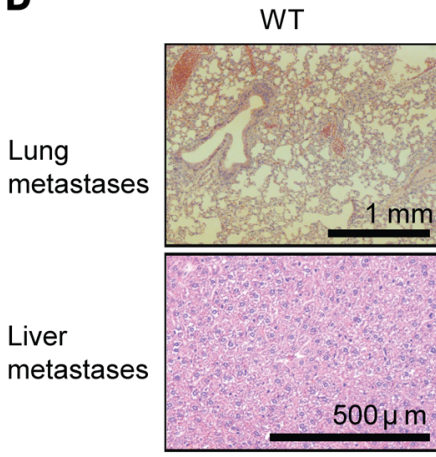

Tumor \#1

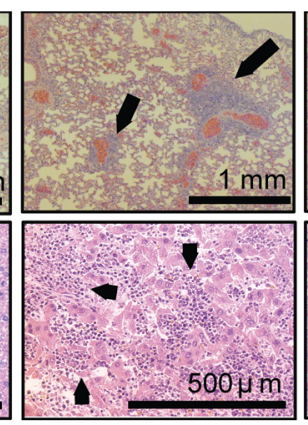

Tumor \#2

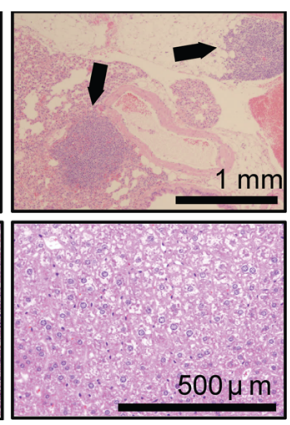

Figure 9. Loss of Beclin 2 increases the incidence of spontaneous lymphoma development. (A) Tumor incidence of WT, Becn2-KO, and Becn2-KO: Map3k3 ${ }^{\triangle M / \Delta M}$ mice at 6 to 36 weeks of age ( $n=45$ in WT group, 38 in Becn2-KO group, and 18 in Becn2-KO:Map3k3 ${ }^{\Delta M / \Delta M}$ group). (B) Photographs and H\&E staining of 2 different tumors from Becn2-KO mice. Red arrows indicate tumors. (C) Photographs of tumors, lymph nodes, spleens, and livers from WT and Becn2-KO mice. (D) H\&E staining of lungs and livers from 2 Becn2-KO mice with lymphomas presented in B. Arrows indicate metastatic tumor nodules in the lung and metastatic tumor cells in the liver. The log-rank test was used for calculating statistical differences in $\mathbf{A}$. ${ }^{*} P<0.05$.

naling of TLRs and their crossregulation with MAPK (JNK, ERK, p38) pathways are not fully understood $(23,24)$; our data suggest that the activation of MEKK3 is differentially regulated by TLR2-, TLR3-, TLR4-, and TLR9-ligand stimulation. Our finding is supported by a previous report showing that MEKK3 interacts with TRAF6 for signaling transduction in response to IL-1 and LPS, but not to CpG, for IL-6 cytokine production (39). Therefore, loss of Beclin 2 results in elevated MEKK3 levels, which may potentially enhance ERK signaling for IL-6 production.

To further understand the mechanisms by which Beclin 2 deficiency increases ERK1/2 and IKK $\alpha / \beta$ signaling, we provide compelling evidence that Beclin 2 targets TAK1 and MEKK3 for autophagic degradation independently of ATG16-LC3 conjugation machinery and Beclin 1. In contrast, ablation of ULK1 or ATG9A could significantly block MEKK3 degradation. Beclin 2-mediated TAK1 and MEKK3 degradation requires ULK1-initiated interaction between Beclin 2 and TAK1/MEKK3 that is ridged by ATG9Avesicles. We further demonstrated that the ULK1 complex (ULK1 and ATG13) was required for Beclin 2-mediated transportation of TAK1/MEKK3-associated ATG9A vesicles to autophagosomes/ lysosomes for degradation, which is supported by the previous finding that ULK1 and ATG13 were required for the recruitment of ATG9A vesicles to PAS for autophagosome formation (26-29). During the ATG16L/LC3B/Beclin 1-independent degradation process of MEKK3, whether other autophagy protein could function redundantly or differently for executing this degradation remained unclear. For example, MEKK3 degradation is partially blocked in ULK1-KO cells, but whether this is due to the ULK2 compensation for the function of ULK1 remains unknown. The LC3/GABARAP family proteins share a high sequence similarity (40), yet could act differently in autophagosome biogenesis. Deficiency in the LC3 subfamily leads to the generation of smaller autophagosomes, whereas deficiency of the GABARAP subfamily leads to the biogenesis of larger autophagosomes (41). Further studies are warranted to determine whether and how other LC3/GABARAP family proteins are involved in the biogenesis of autophagosomes to compensate for the loss of LC3B during autophagic degradation of MEKK3. Although LPS has been reported as inducing autophagy through TRIF-dependent TLR4 signaling (42), our results show that neither the interaction between ATG9A and MEKK3 nor the MEKK3 protein level was affected by LPS stimulation, suggesting that LPS-induced TLR signaling may not be required in the Beclin 2-mediated autophagic degradation of MEKK3.

SNARE or RAB GTPase family proteins have been reported as playing a critical role in membrane fusion in autophagy (25, 30). STX5 and STX6 are 2 target-SNAP receptors (t-SNAREs) serving for specific vesicle docking and fusion. STX5 and STX6 have been reported as driving the fusion of autophagosomes with lysosomes and regulating the fusion between endosomes and autophagosomes, respectively $(43,44)$. Our data show that 
A

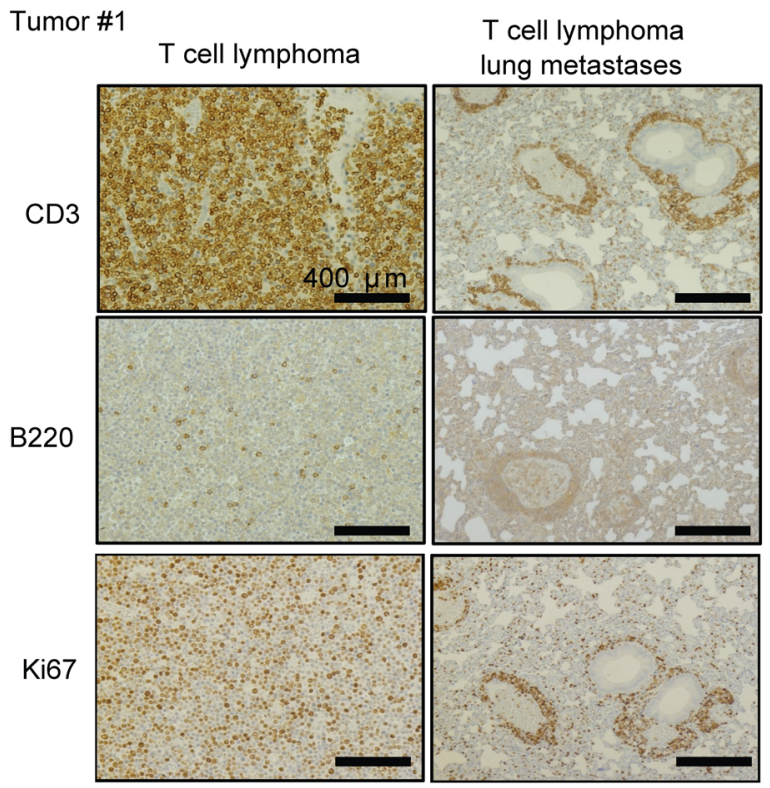

Tumor \#2/B cell lymphoma lung metastasis

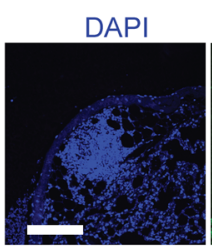

DAPI

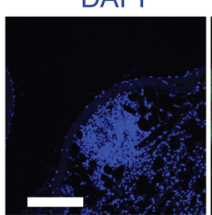

B220

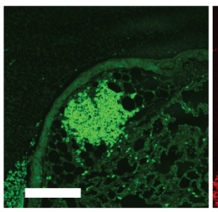

CD3

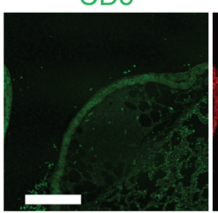

Ki67

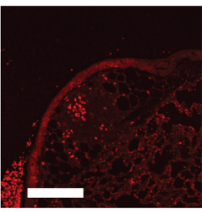

Ki67

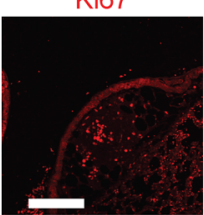

Merge

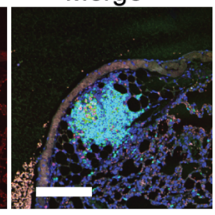

Merge

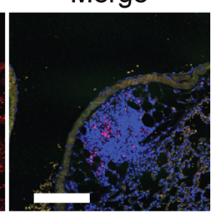

B

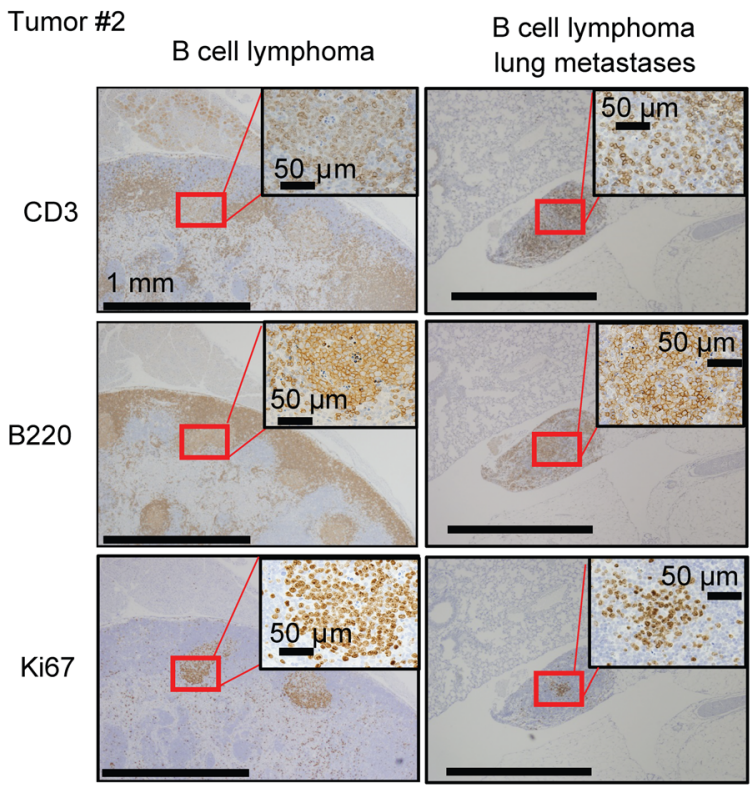

Tumor \#2/B cell lymphoma

DAPI

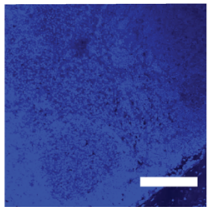

DAPI

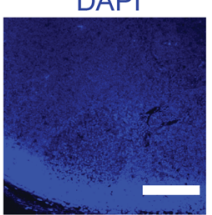

B220

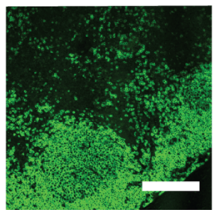

CD3

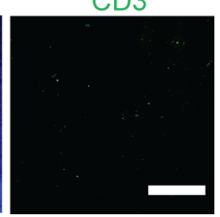

Ki67

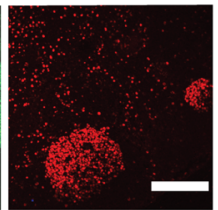

Ki67

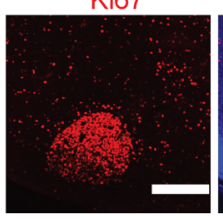

Merge

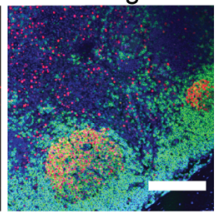

Merge

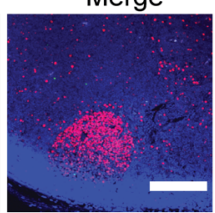

Figure 10. Lymphoma-bearing Becn2-KO mice could develop metastatic nodules in the lung and liver. (A and B) Immunohistochemical staining of CD3, B220, or Ki67 in lymphoma and lung samples from tumors no. 1 (A) and no. 2 (B). (C) Representative confocal images of lymphoma and lung tissue from tumor no. 2 stained with antibodies against CD3, B220, and Ki67 followed by FITC- and Alexa Fluor 555-conjugated secondary antibodies. Scale bars: $250 \mu \mathrm{m}$.

Beclin 2 could interact with STX5 and STX6, both of which are required for Beclin 2-mediated MEKK3-associated ATG9A vesicle fusion to phagophores for autophagic degradation. Although a previous report showed that cells deficient in STX5 have compromised cathepsin B activity (44), we did not detect any significant changes in lysosomal degradation or cathepsin B activity among WT, STX5-KO, or STX6-KO cells (data not shown). Overall, we show 3 lines of evidence to support the molecular mechanisms of Beclin 2-mediated MEKK3 degradation through an ATG9-dependent, but ATG16L/LC3/Beclin 1-independent, autophagic pathway (Supplemental Figure 7E): (a) both Beclin 2 and ATG9A interact with MEKK3 and ULK1, but not with other ULK1 complex subunits (ATG13, FIP200, or ATG101); ULK1 KO or ATG13 KD markedly, but not completely, blocks MEKK3 degradation; (b) the interaction between Beclin 2 and MEKK3 requires ULK complex initiation and ATG9A engagement; ATG9A KO completely blocks Beclin 2-mediated MEKK3 degradation; and (c) Beclin 2 interacts with STX5/6 to promote the fusion of MEKK3-associ- ated ATG9A vesicles with phagophores for MEKK3 degradation; loss of STX5/6 blocks Beclin 2-mediated MEKK3 degradation due to the failure of this fusion.

Consistent with these in vitro observations, deletion of Map3k3 completely rescued the proinflammatory phenotypes (such as splenomegaly and lymphadenopathy) observed in Becn2deficient mice, while ablation of Map3k7 partially rescued these phenotypes. The critical role of MEKK3 in NF- $\kappa \mathrm{B}$ activation has been reported through direct phosphorylation of $I K K \alpha / \beta$ upon induction with TNF- $\alpha$ (45). However, we previously showed that ablation of Map3k7 (TAK1) in myeloid lineage enhanced the NF- $\kappa B$ and p38 MAP kinase activation in neutrophils and induced splenomegaly and lymphadenopathy in mice, while specific deletion of Map3k3 (MEKK3) partially reduced the splenomegaly observed in Map $3 k 7^{\Delta \mathrm{M} / \Delta \mathrm{M}}$ mice (15). Therefore, the findings that myeloid-specific ablation of $M a p 3 k 3$, but not $M a p 3 k 7$, rescued the phenotypes in Becn2-KO mice were consistent with our previous study (15). Collectively, we demonstrated that MEKK3 played a dominant role in the 
A

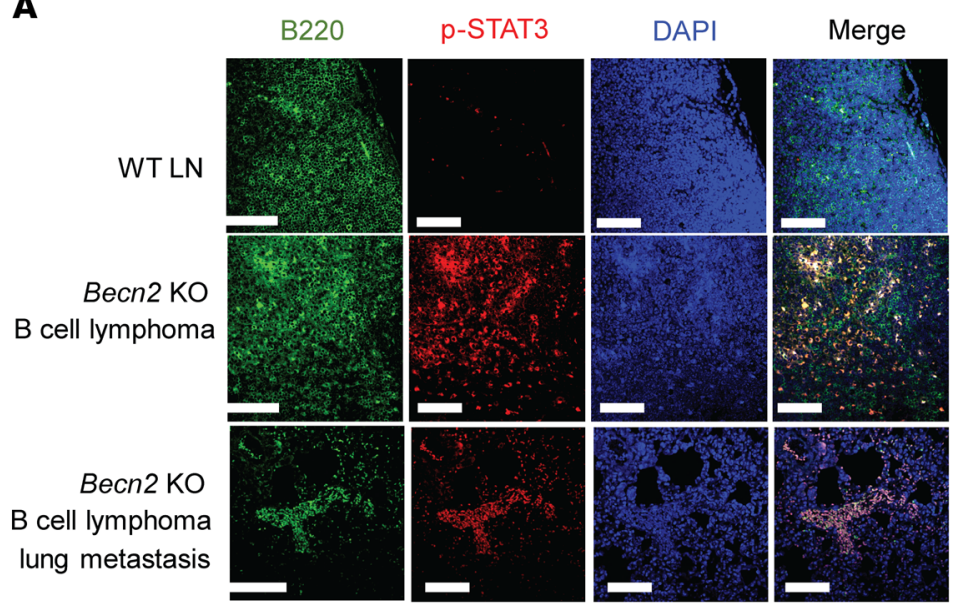

B

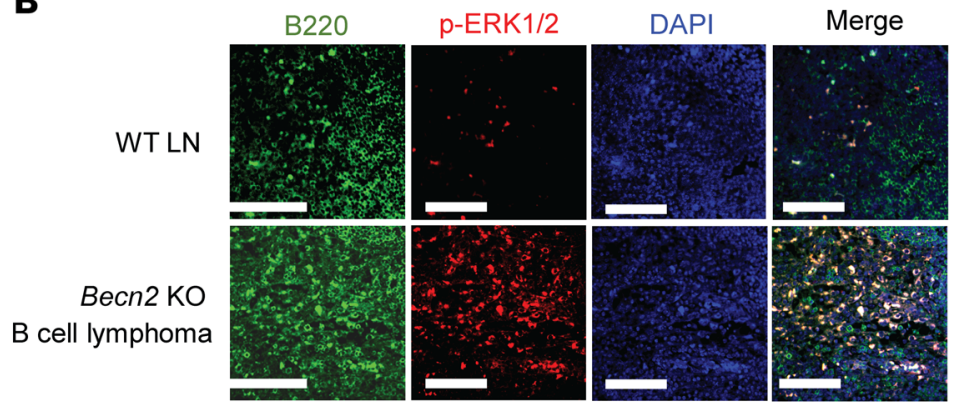

D

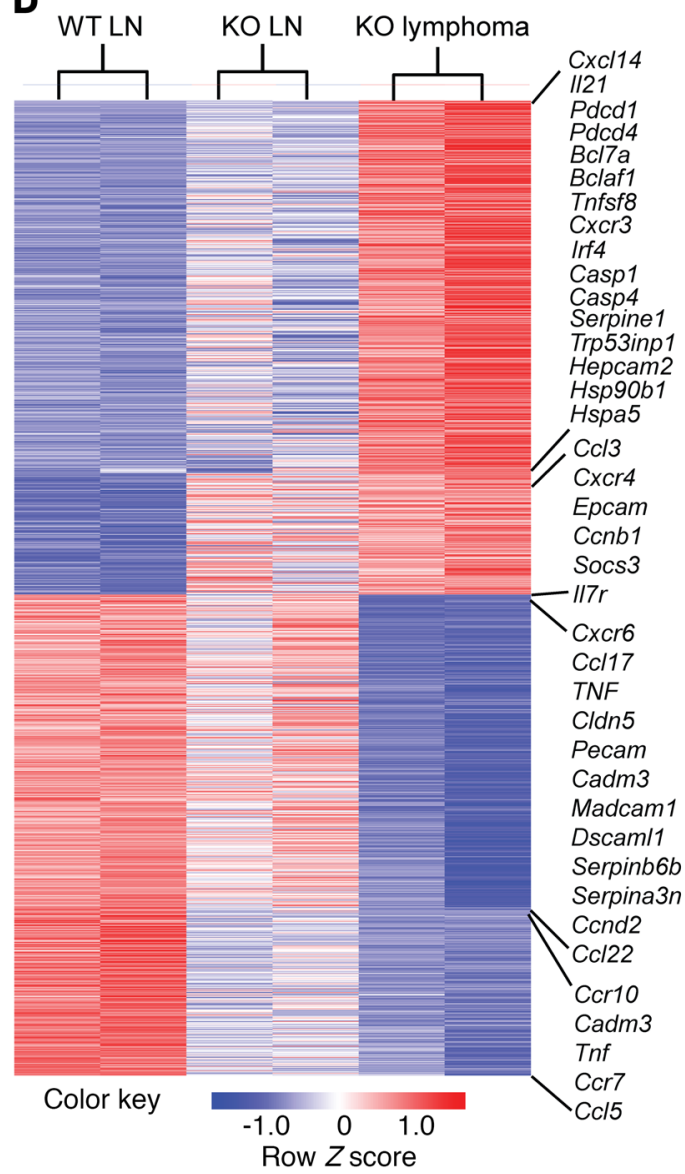

C

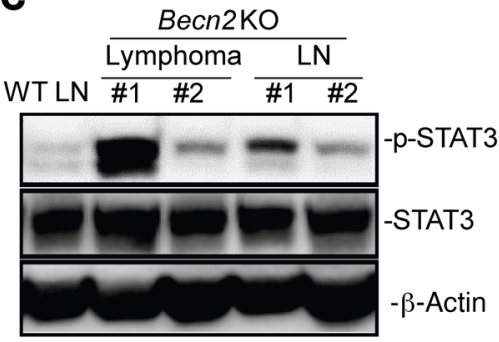

E

KEGG pathway

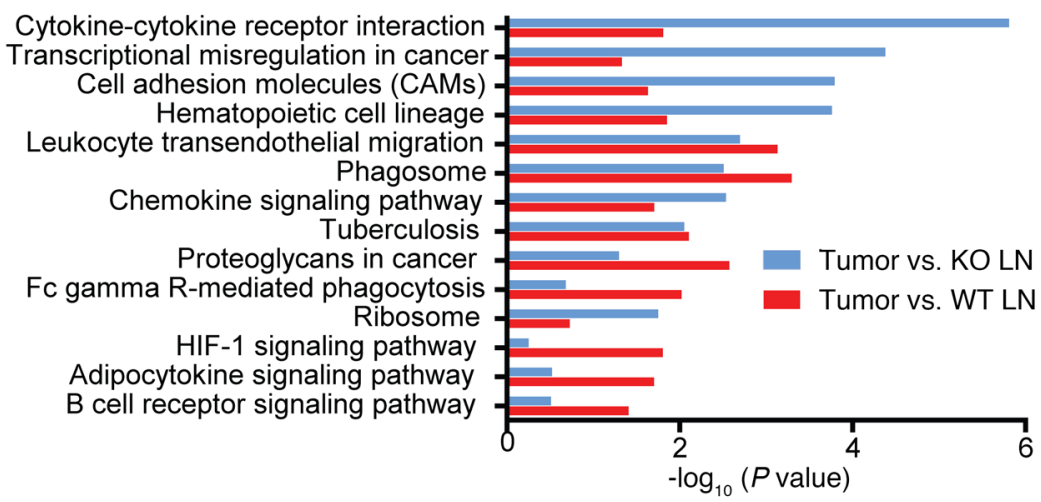

Figure 11. STAT3 activation and cytokine/chemokine expression in Becn2-KO lymphoma. (A) Confocal images of Becn2-KO lymphomas, lymphomaderived lung metastases, and WT lymph nodes stained with antibodies against B220 and p-STAT3 followed by FITC- or Alexa Fluor 555-conjugated secondary antibodies. Scale bars: $250 \mu \mathrm{m}$. (B) Confocal images of Becn2-KO lymphomas and WT lymph nodes stained with antibodies against B220 and p-ERK1/2, followed by labeling with FITC- or Alexa Fluor 555-conjugated secondary antibodies. Scale bars: $250 \mu \mathrm{m}$. (C) Immunoblot analysis of p-STAT3 and STAT3 expression in lymph nodes or lymphomas from WT and Becn2-KO mice. (D) Heatmap of the DEGs in Becn2-KO lymphoma samples compared with WT or Becn2-KO lymph nodes. The most significant DEGs involved in STAT3 signaling, cytokine-cytokine receptor interaction, chemokine signaling pathways, and cell-cell adhesion are listed on the right side. (E) Significantly enriched KEGG pathways in Becn2-KO lymphomas compared with WT or Becn2-KO lymph nodes.

control of ERK1/2 signaling, proinflammatory cytokine production, splenomegaly, and lymphadenopathy in Becn2-KO mice.

Heterozygous deletion of Becn1 in mice increases the incidence of spontaneous tumors $(35,46)$, suggesting that $B e c n 1$ is a haploinsufficient tumor suppressor gene. Heterozygous deletion of Becn2 leads to defective autophagy, obesity, and insulin resistance (8). However, it is unclear whether the ablation of Becn2 results in tumor development. Our study provides compelling evidence that homozygous $\mathrm{KO}$ of Becn2 increases the incidence of spontaneous B and T cell lymphomas, which is consistent with 
A

ㅁ Q-PCR RNAseq $B c / 7 a$

\section{帘}
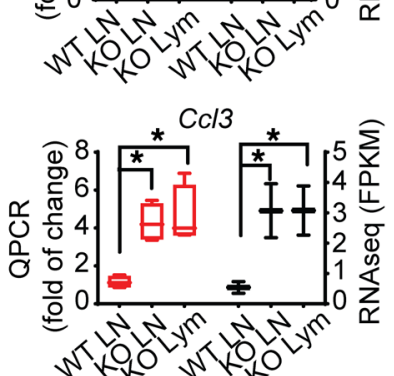
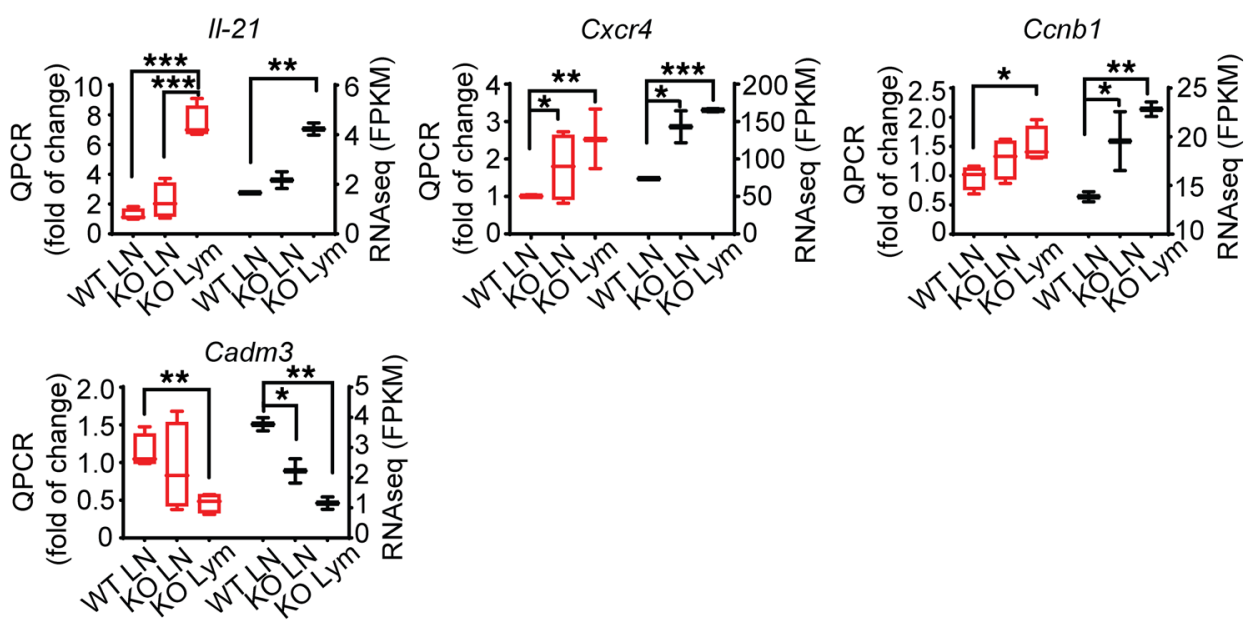

B
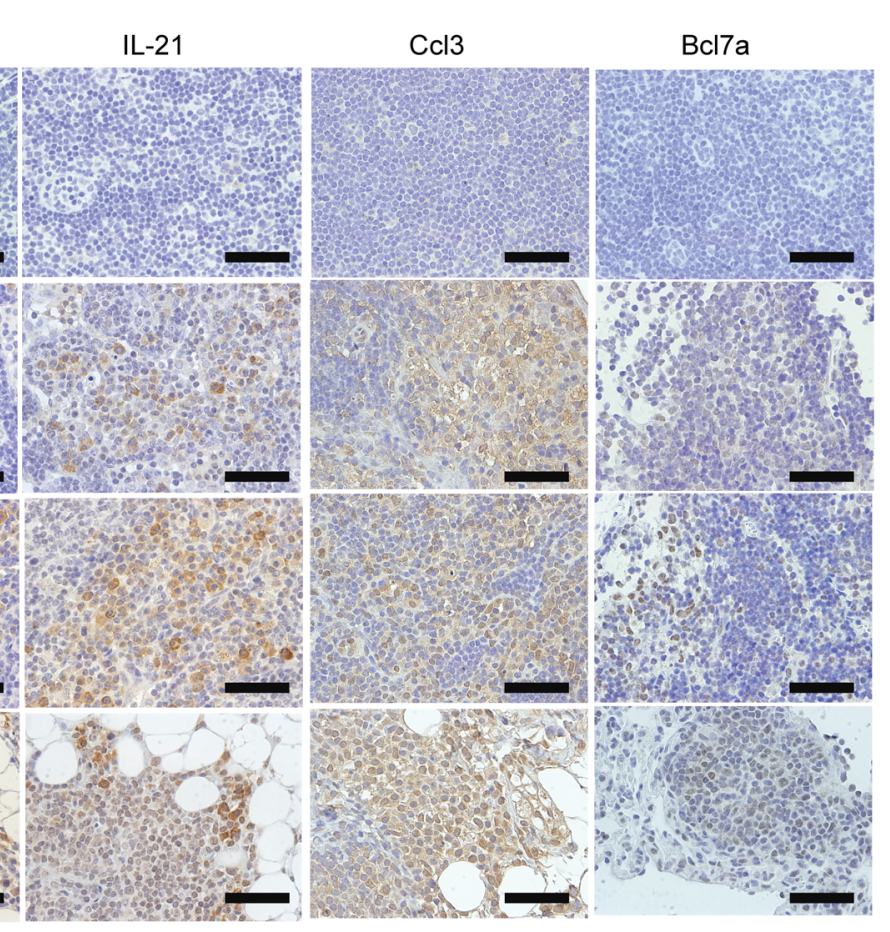

Figure 12. Protumorigenic cytokine, chemokine, and oncogene expression in Becn2-KO lymphoma. (A) Real-time PCR analysis for mRNA levels of the indicated genes in WT Iymph nodes, Becn2-KO lymph nodes, and Becn2-KO Iymphomas (4 biological replicates for each group) compared with the fragments per kilobase of transcript per million mapped reads (FPKM) value of each gene from RNA-Seq analysis. Whisker boxes represent median (center line), and upper and lower lines represent lowest to highest value. (B) Immunohistochemical staining of lymphomas and lung metastases using antibodies against Cxcr4, IL-21, Bcl-7a, and Ccl3. Scale bars: $100 \mu \mathrm{m}$. Statistical differences between groups were calculated using 1-way ANOVA with Tukey's multiple comparison test $(\mathbf{A}) .{ }^{*} P<0.05 ;{ }^{* *} P<0.01 ;{ }^{* *} P<0.001$.

the hyperproliferation of $\mathrm{T}$ and $\mathrm{B}$ cells in peripheral lymphoid tissues, the elevated proinflammatory cytokines, and the enhanced ERK and STAT3 signaling in Becn2-deficient mice. Beclin 1 has been shown to interact with antiapoptotic Bcl-2 family members via its BH3 domain and restrain tumorigenesis through Mcl-1 destabilization (36). In contrast, we show that loss of Beclin 2 neither increased the expression of Bcl-2 or Mcl-1 nor increased the phosphorylation of Bcl-2. Instead, the persistent activation of STAT3 in immune cells and tumor tissues, in concert with the elevated IL-6 production, plays a critical role in inflammatory signaling and tumor development in Becn2-deficient mice. Elevated STAT3 and ERK1/2 signaling pathways have been reported as playing important roles in initiating a premetastatic tumor niche and promoting tumor development and metastasis $(11,24$, $33,47)$. Our RNA-Seq analysis further supports the notion that the lymphoma development in Becn2-deficient mice is associated with persistent activation of STAT3 signaling and increased expression of protumorigenic cytokines, chemokines, and oncogenes. In contrast, cell-cell junction and adhesion molecules are downregulated in Becn2-deficient mice, thereby facilitating 
$\mathbf{A}$
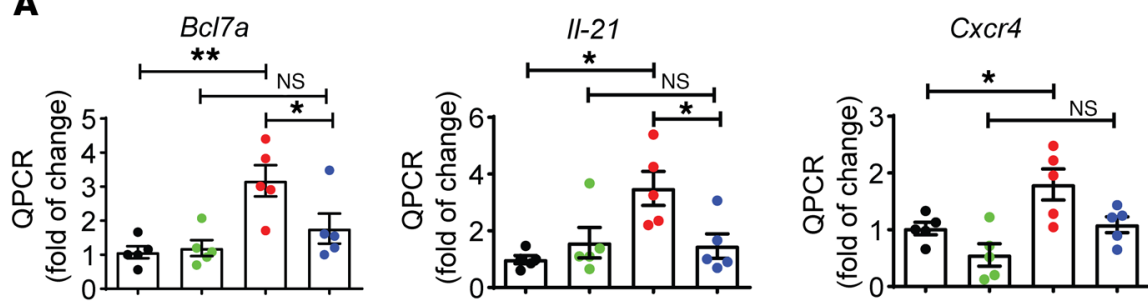

- $W T+C \operatorname{trl} A b$

- $\mathrm{WT}+\mathrm{IL}-6 \mathrm{Ab}$

- Becn2 KO + Ctrl Ab

- Becn2 KO + IL-6 Ab
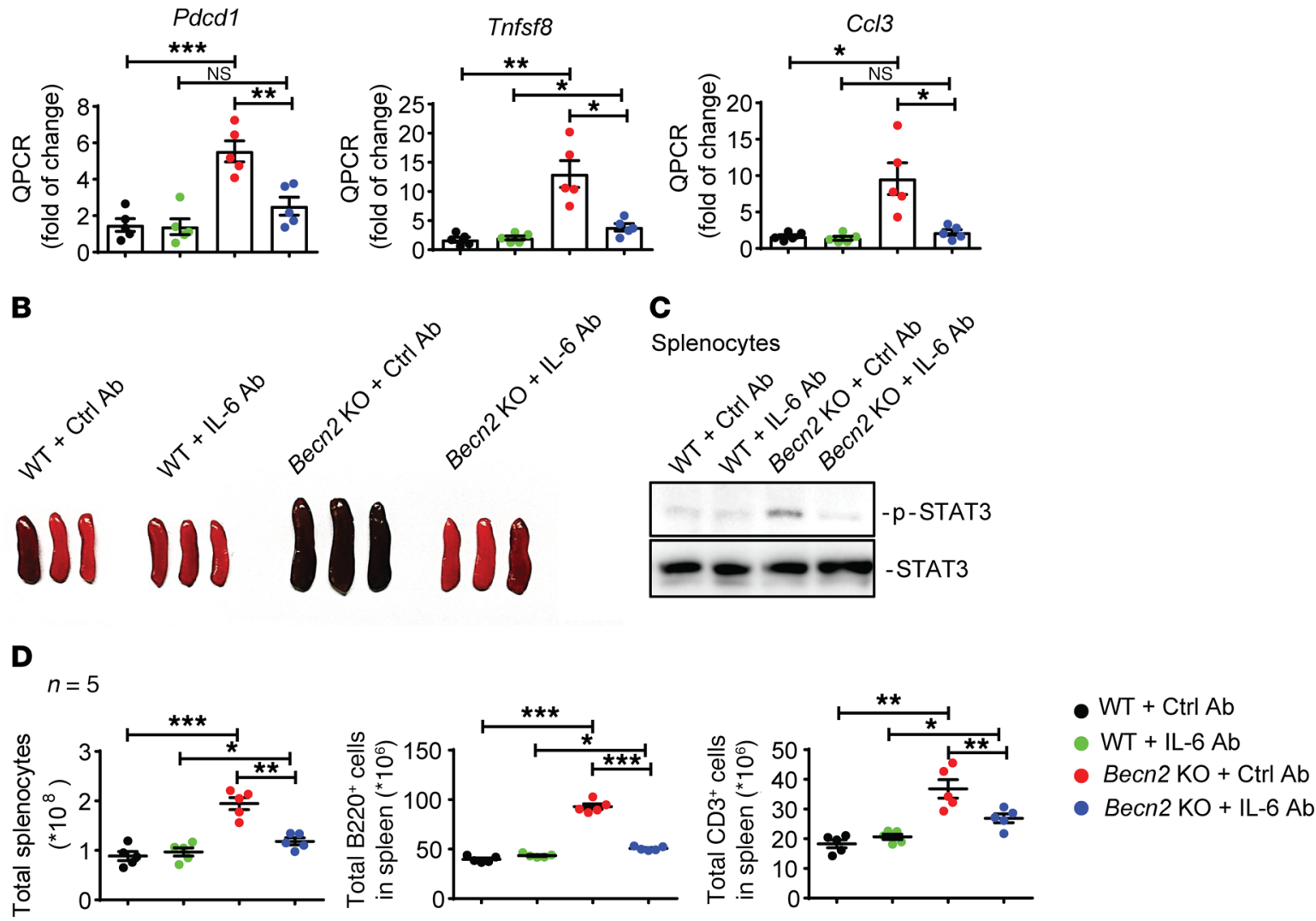

Figure 13. IL-6 neutralizing antibody treatment rescues phenotypes observed in Becn2-KO mice. (A) Real-time PCR analysis for mRNA levels of the indicated genes in lymph nodes from WT or Becn2-KO mice receiving control or IL-6 neutralizing antibody treatment $(n=5$, i.p., $200 \mu \mathrm{g} / \mathrm{mouse}$, every other day for 4 consecutive weeks). (B) Spleens of WT and Becn2-KO mice receiving control or IL-6 neutralizing antibody treatment $(n=5)$. (C) Immunoblot analysis of p-STAT3 in splenocytes from indicated groups. Representative images of 3 independent Western blots. (D) Total numbers of splenocytes and flow cytometry-based quantification of $\mathrm{B}_{220^{+}}$and $\mathrm{CD}^{+}$cell populations in spleens counted from mice of indicated groups $(n=5)$. Statistical differences between groups were calculated using 1-way ANOVA with Tukey's multiple comparison test (A and $\mathbf{D})$. ${ }^{*} P<0.05 ;{ }^{*} P<0.01 ;{ }^{* * *} P<0.001$.

tumor invasion and metastasis. By using IL-6 neutralizing antibody to treat Becn2-KO mice, the levels of the upregulated key genes identified in Becn2-KO lymphoma, the levels of p-STAT3, and the total numbers of splenocytes and lymphocytes were all markedly reduced compared with those in Becn2-KO mice treated with a control antibody, suggesting the important role of IL-6 production in the promotion of lymphoma development in Becn2$\mathrm{KO}$ mice. Based on these findings, we propose a working model to illustrate how Beclin 2 controls the stability of MEKK3 and TAK1 through an ATG9-dependent, but ATG16L/LC3/Beclin 1independent, autophagic pathway, thus regulating ERK and IKK signaling-mediated IL- 6 production, which in turn activates the STAT3 signaling pathway. The activation of ERK and IL-6-STAT3 signaling further promotes tumor development and metastasis in Becn2-KO mice. Our results have identified an important role of Beclin 2 in innate immune signaling and tumor development, thus providing potential therapeutic targets for the prevention and treatment of cancer.

\section{Methods}

All RNA-Seq data that support the findings of this study were deposited in the NCBI's Gene Expression Omnibus database (GEO GSE111539).

Statistics. Statistical analyses were performed using GraphPad Prism, version 6.0, and Excel, with a minimum of 3 biological independent samples for significance. A log-rank test was used for mouse survival or tumor incidence analysis. A 1-way ANOVA or unpaired 2-tailed Student's $t$ test was applied for other comparisons. $P$ values of less than 0.05 were considered statistically significant.

Study approval. Animal experiments in this study were approved and carried out following the protocol (AUP-0115-0005 and 
AUP-0618-0036) provided by the IACUC at Houston Methodist Research Institute. The IACUC uses the NIH Guide for the Care and Use of Laboratory Animals (National Academies Press, 2011), which is based on US Government Principles for Utilization and Care of Vertebrate Animals Used in Testing, Research, and Training (National Academies Press, 2011).

Supplemental Methods can be found with this article online.

Information regarding reagents, commercially available kits, and plasmids used in this study is available in Supplemental Table 1. Oligo sequences for gene-knockout, genotyping, and real-time PCR are listed in Supplemental Table 2. Full, uncut gels are shown in the supplemental material

\section{Author contributions}

$\mathrm{MZ}$ and RFW designed the experiments and analyzed data. MZ, CG, PT, GD, CX, BN, BY, Y Zhang, CL, GN, Y Zhao, and HYW performed experiments and provided suggestions. $\mathrm{KC}, \mathrm{CJ}$, and $\mathrm{MZ}$ analyzed the RNA-Seq data. BL provided Becn2-deficient mice. MZ, $\mathrm{CX}$, and RFW wrote the paper. RFW supervised the entire study.

\section{Acknowledgments}

This work was in part supported by grants from the National Cancer Institute, NIH (R01CA101795 and U54CA210181), and startup funds from Houston Methodist Research Institute and University of Southern California (to RFW) as well as by a Norris Comprehensive Cancer Center grant (P30CA014089). We thank the Houston Methodist Immunomonitoring Core for help with the CyTOF experiments and data analysis.

Address correspondence to: Rong-Fu Wang, 1450 Biggy St., NRT5503, Los Angeles, California 90033, USA. Phone: 323.442.7981; Email: rongfuwa@usc.edu. Or to: Guangjun Nie, Beiyitiao 11, Zhongguancun, Beijing 100190, China. Phone: 86.10.82545638; Email: niegj@nanoctr.cn.
1. Shibutani ST, Saitoh T, Nowag H, Münz C, Yoshimori T. Autophagy and autophagy-related proteins in the immune system. Nat Immunol. 2015;16(10):1014-1024.

2. Feng Y, He D, Yao Z, Klionsky DJ. The machinery of macroautophagy. Cell Res. 2014;24(1):24-41.

3. Codogno P, Mehrpour M, Proikas-Cezanne T. Canonical and non-canonical autophagy: variations on a common theme of self-eating? Nat Rev Mol Cell Biol. 2011;13(1):7-12.

4. Martinez-Martin N, et al. A switch from canonical to noncanonical autophagy shapes B cell responses. Science. 2017;355(6325):641-647.

5. Henault J, et al. Noncanonical autophagy is required for type I interferon secretion in response to DNA-immune complexes. Immunity. 2012;37(6):986-997.

6. Green DR, Galluzzi L, Kroemer G. Mitochondria and the autophagy-inflammation-cell death axis in organismal aging. Science. 2011;333(6046):1109-1112.

7. Heckmann BL, et al. LC3-associated endocytosis facilitates $\beta$-amyloid clearance and mitigates neurodegeneration in murine Alzheimer's disease. Cell. 2019;178(3):536-551.e14.

8. He C, et al. Beclin 2 functions in autophagy, degradation of $G$ protein-coupled receptors, and metabolism. Cell. 2013;154(5):1085-1099.

9. Dong X, et al. Endolysosomal trafficking of viral $G$ protein-coupled receptor functions in innate immunity and control of viral oncogenesis. Proc Natl Acad Sci USA. 2016;113(11):2994-2999.

10. Crunkhorn S. Metabolic disorders: Breaking the links between inflammation and diabetes. Nat Rev Drug Discov. 2013;12(4):261.

11. Park EJ, et al. Dietary and genetic obesity promote liver inflammation and tumorigenesis by enhancing IL- 6 and TNF expression. Cell. 2010;140(2):197-208.

12. Levine B, Mizushima N, Virgin HW. Autophagy in immunity and inflammation. Nature. 2011;469(7330):323-335.

13. Deretic V, Saitoh T, Akira S. Autophagy in infection, inflammation and immunity. Nat Rev Immunol. 2013;13(10):722-737.

14. Zhong Z, Sanchez-Lopez E, Karin M. Autoph- agy, inflammation, and immunity: a troika governing cancer and its treatment. Cell. 2016;166(2):288-298.

15. Ajibade AA, et al. TAK1 negatively regulates NF- $\mathrm{\kappa B}$ and $\mathrm{p} 38$ MAP kinase activation in $\mathrm{Gr}-1+\mathrm{CD} 11 \mathrm{~b}+$ neutrophils. Immunity. 2012;36(1):43-54.

16. Yu X, et al. Cross-regulation of two type I interferon signaling pathways in plasmacytoid dendritic cells controls anti-malaria immunity and host mortality. Immunity. 2016;45(5):1093-1107.

17. Cadwell K. Crosstalk between autophagy and inflammatory signalling pathways: balancing defence and homeostasis. Nat Rev Immunol. 2016;16(11):661-675.

18. Iwasaki A, Medzhitov R. Control of adaptive immunity by the innate immune system. Nat Immunol. 2015;16(4):343-353.

19. Jia K, et al. Autophagy genes protect against Salmonella typhimurium infection and mediate insulin signaling-regulated pathogen resistance. Proc Natl Acad Sci USA. 2009;106(34):14564-14569.

20. Shelly S, Lukinova N, Bambina S, Berman A, Cherry S. Autophagy is an essential component of Drosophila immunity against vesicular stomatitis virus. Immunity. 2009;30(4):588-598.

21. Saitoh T, et al. Loss of the autophagy protein Atg16L1 enhances endotoxin-induced IL-1beta production. Nature. 2008;456(7219):264-268.

22. Zhang Q, Lenardo MJ, Baltimore D. 30 years of NF-kB: a blossoming of relevance to human pathobiology. Cell. 2017;168(1-2):37-57.

23. Oeckinghaus A, Hayden MS, Ghosh S. Crosstalk in NF- $\mathrm{BB}$ signaling pathways. Nat Immunol. 2011;12(8):695-708.

24. Arthur JS, Ley SC. Mitogen-activated protein kinases in innate immunity. Nat Rev Immunol. 2013;13(9):679-692.

25. Nishida Y, et al. Discovery of Atg5/Atg7-independent alternative macroautophagy. Nature. 2009;461(7264):654-658.

26. Zhou C, et al. Regulation of mATG9 trafficking by Src- and ULK1-mediated phosphorylation in basal and starvation-induced autophagy. Cell Res. 2017;27(2):184-201.

27. Suzuki SW, et al. Atg13 HORMA domain recruits
Atg9 vesicles during autophagosome formation. Proc Natl Acad Sci USA. 2015;112(11):3350-3355.

28. Rao Y, Perna MG, Hofmann B, Beier V, Wollert T. The Atg1-kinase complex tethers Atg9-vesicles to initiate autophagy. Nat Commun. 2016;7:10338.

29. Yamamoto $\mathrm{H}$, et al. Atg9 vesicles are an important membrane source during early steps of autophagosome formation. J Cell Biol. 2012;198(2):219-233.

30. Tsuboyama K, Koyama-Honda I, Sakamaki Y, Koike M, Morishita H, Mizushima N. The ATG conjugation systems are important for degradation of the inner autophagosomal membrane. Science. 2016;354(6315):1036-1041.

31. Lamb CA, Yoshimori T, Tooze SA. The autophagosome: origins unknown, biogenesis complex. Nat Rev Mol Cell Biol. 2013;14(12):759-774.

32. Nair U, et al. SNARE proteins are required for macroautophagy. Cell. 2011;146(2):290-302.

33. Liang J, et al. Sphingosine-1-phosphate links persistent STAT3 activation, chronic intestinal inflammation, and development of colitis-associated cancer. Cancer Cell. 2013;23(1):107-120.

34. Yu H, Lee H, Herrmann A, Buettner R, Jove R. Revisiting STAT3 signalling in cancer: new and unexpected biological functions. Nat Rev Cancer. 2014;14(11):736-746.

35. Gong $C$, et al. Beclin 1 and autophagy are required for the tumorigenicity of breast cancer stem-like/progenitor cells. Oncogene. 2013;32(18):2261-2272-2272e.1.

36. Elgendy $\mathrm{M}$, et al. Beclin 1 restrains tumorigenesis through Mcl-1 destabilization in an autophagyindependent reciprocal manner. Nat Commun. 2014;5:5637.

37. Nakahira K, et al. Autophagy proteins regulate innate immune responses by inhibiting the release of mitochondrial DNA mediated by the NALP3 inflammasome. Nat Immunol. 2011;12(3):222-230.

38. Reeves MB, Compton T. Inhibition of inflammatory interleukin- 6 activity via extracellular signal-regulated kinase-mitogen-activated protein kinase signaling antagonizes human cytomegalovirus reactivation from dendritic cells. J Virol. 2011;85(23):12750-12758. 
39. Huang $Q$, et al. Differential regulation of interleukin 1 receptor and Toll-like receptor signaling by MEKK3. Nat Immunol. 2004;5(1):98-103.

40. Schaaf MB, Keulers TG, Vooijs MA, Rouschop KM. LC3/GABARAP family proteins: autophagy-(un)related functions. FASEB J. 2016;30(12):3961-3978.

41. Weidberg H, Shvets E, Shpilka T, Shimron F, Shinder V, Elazar Z. LC3 and GATE-16/GABARAP subfamilies are both essential yet act differently in autophagosome biogenesis. $E M B O$
J. 2010;29(11):1792-1802.

42. Xu Y, Jagannath C, Liu XD, Sharafkhaneh A, Kolodziejska KE, Eissa NT. Toll-like receptor 4 is a sensor for autophagy associated with innate immunity. Immunity. 2007;27(1):135-144.

43. Nozawa T, Minowa-Nozawa A, Aikawa C, Nakagawa I. The STX6-VTI1B-VAMP3 complex facilitates xenophagy by regulating the fusion between recycling endosomes and autophagosomes. Autophagy. 2017;13(1):57-69.

44. Renna M, et al. Autophagic substrate clearance requires activity of the syntaxin-5 SNARE complex. J Cell Sci. 2011;124(Pt 3):469-482.

45. Yang J, et al. The essential role of MEKK 3 in TNF-induced NF-kappaB activation. Nat Immunol. 2001;2(7):620-624.

46. $\mathrm{Qu} \mathrm{X}$, et al. Promotion of tumorigenesis by heterozygous disruption of the beclin 1 autophagy gene. J Clin Invest. 2003;112(12):1809-1820.

47. Dhillon AS, Hagan S, Rath O, Kolch W. MAP kinase signalling pathways in cancer. Oncogene. 2007;26(22):3279-3290. 